\title{
On the identification of the axial force and bending stiffness of stay cables anchored to flexible supports
}

\author{
Francesco Foti ${ }^{\mathrm{a}, *}$, Margaux Geuzaine ${ }^{\mathrm{b}, \mathrm{c}}$, Vincent Denö̈l ${ }^{\mathrm{b}}$ \\ ${ }^{a}$ Department of Civil and Environmental Engineering, Politecnico di Milano, \\ P.zza Leonardo da Vinci 32, 20133 Milano, Italy \\ ${ }^{b}$ Structural Engineering Division, Faculty of Applied Sciences, University of Liège, \\ Allée de la Découverte 1, 4000 Liège, Belgium \\ ${ }^{c}$ F.R.S.-FNRS, National Fund for Scientific Research, Belgium
}

\begin{abstract}
The generic model of a cable with small bending stiffness and anchored to flexible supports in rotation and translation is considered. An asymptotic analysis of the natural frequencies of this generic model is derived and shows that, for small bending stiffness, the first few natural frequencies can be expressed as a function of the cable axial force, the small bending stiffness and a single dimensionless group collecting the information of all other problem parameters. This formulation is used to develop an identification procedure of the cable axial force. Two formulations are proposed, one numerical and one semi-analytical based on a simple linear regression model. Both methods do not attempt at separately identifying the problem parameters since the observability analysis has revealed that only the cable axial force, the bending stiffness and the dimensionless group can be identified. In particular, the second method is very simple to implement and provides estimates of the cable axial force which account for the flexibility of the support. The proposed method can therefore be seen as an extension of usual identification techniques based on linear regressions of natural frequencies vs. mode number relations, by considering at the same time the bending stiffness and the deformability of supports. Being simple and robust as shown by means of an uncertainty quantification analysis, the proposed method can be conveniently embedded in the framework of a continuous monitoring strategy.
\end{abstract}

Keywords: Stay cables, Axial force, Bending stiffness, Parameter identification, Structural health monitoring, Differential Evolution

\section{Introduction}

The identification of cable axial force is of paramount importance for structural health monitoring and safety assessment of stayed bridges [44] and other special structures, such as large-span cable roofs [8]. Experimental testing campaigns, hence, are typically carried out both at early construction stages, to check the compliance of the cable axial force with design requirements, and during the service life of the structure. Monitoring variations in time of the axial force, indeed, can allow for early detection of potentially harmful damage phenomena $[44,36,20,52,39]$.

Direct measurements of the axial force in stay cables can be obtained through permanent installation of load cells or by means of lift-off tests performed with hydraulic jacks. Both testing techniques require expensive instrumentation. Moreover, lift-off tests are potentially dangerous and need to be executed with great care, since they involve removal of portions of the anchoring system [35, 11, 24]. On the other hand, indirect measurements based on both static (e.g. [47]) and dynamic (e.g. $[17,6,7])$ testing can be effectively used to get axial force estimates.

\footnotetext{
* Corresponding author

Email addresses: francesco.foti@polimi.it (Francesco Foti), mgeuzaine@uliege.be (Margaux Geuzaine), v.denoel@uliege.be (Vincent Denoël)
} 
Dynamic testing techniques have been thoroughly investigated in the past decades and are nowadays widely employed in practice, since they can be performed in operational conditions and provide the ground for quick and cheap structural parameter identification procedures $[25,4,13$, $58,40]$.

Vibration-based identification procedures typically rely on the knowledge of (i) a set of experimentally determined natural frequencies of the stay cable, and (ii) a mechanical model that relates the natural frequencies to the axial force value. Reliability of results, hence, is inherently affected by the predictive capabilities of the underlying mechanical model.

Due to their slenderness and inherent flexibility, structural cables are often modeled as "perfectly flexible" one-dimensional continua, that can only withstand axial forces. The dynamic behavior of perfectly flexible cable models has been widely studied by accounting for the effects of both geometric and elastic stiffness terms (see e.g. [31, 56, 50]).

Small vibrations of shallow cables, i.e. suspended cables characterized by small values of the sag-to-span ratio $\delta$ (in the order of $\delta<\frac{1}{8}$ ), have been thoroughly investigated by Irvine and Caughey $[31,30]$ under the assumption of quasi-static stretching. A key feature of the linearized model derived by Irvine and Caughey is the decoupling between in-plane and out-of-plane vibrations, i.e. between the components of motion respectively belonging to and perpendicular to the plane of the gravity loads. In-plane spectral properties are shown to be governed by a single nondimensional parameter $\lambda^{2}$ that can be conveniently regarded as a characteristic ratio of the elastic to the geometric stiffness terms. Small values of the Irvine's parameter $\lambda^{2}$ (i.e. $\lambda^{2} \lesssim 1$ ) are related to cables strung at relatively high values of axial force and with a small sag-to-span ratio, such as the ones typically employed as stays (see e.g. [6]). It is worth noting that, for vanishing values of the parameter $\lambda^{2}$, both the effect of the elastic deformation (i.e. the cable extensibility) and of the static curvature of the cable (i.e. the cable sagging) become negligible with respect to the geometric stiffness contribution and the in-plane linearized behavior of the cable tends the one of the well-known unstreatchable taut string model. The small out-of-plane vibrations of the shallow cable model, on the other hand, turns out to be always governed by the taut string equation of motion [31].

A generalization of the model of Irvine and Caughey, that fully accounts for the transition from shallow to deeply non-shallow cable profiles has been proposed by Lacarbonara et al. [37] and served as the basis to a thorough investigation of the non-linear free vibrations of suspended cables [38]. Excellent reviews on the non-linear deterministic and stochastic dynamics of perfectly flexible cables have been provided, respectively, by Rega [50, 51] and Ibrahim [29].

Enriched cable models, accounting for bending and torsional stiffness terms, have also been proposed in the literature to correctly represent some characteristic features of the static and dynamic response of structural cables, such as the geometric coupling between torsional and bending behavior [3] or between axial and torsional behavior [43], and the hysteretic bending behavior of metallic cables [21]. Moreover, accounting for these "beam-like" stiffness terms is of pivotal importance for the characterization of the stress-strain state within the boundary layers that can occur in the neighborhood of the constraints $[1,19,18]$.

Small planar vibrations of taut cables (with sag-to-span ratio $\delta<<1$ ) characterized by small values of the Irvine's parameter $\lambda^{2}$ are often investigated by resorting to the classic unstreatchable Euler-Bernoulli beam model [6]. As it has been clearly shown by Arena et al. [1], however, the Euler-Bernoulli model can be inaccurate whenever in presence of significant torsional effects or static configurations characterized by values of the sag-to-span ratio in the order of $\delta=1 / 15$ or greater. In these cases, a general formulation based on three-dimensional geometrically exact curved rod models should be preferred (e.g. [1, 41]).

Different modeling assumptions have been adopted in the literature on stay cable axial force identification problems, including: (a) the well-known taut-string model [12, 25, 4], (b) cable models accounting for the bending stiffness, but neglecting cable sagging and axial extensibility effects $[2,9,24,28]$, and (c) cable models accounting for bending stiffness, cable sagging and axial extensibility effects $[34,42,45,62]$. Within this context, it has been clearly highlighted that cable sagging and axial extensibility play a negligible role on the in-plane dynamics of stay cables characterized by small values of the sag-to-span ratio, often encountered in practice (e.g. 
$[31,45,6])$. Neglecting bending stiffness effects, on the other hand, can lead to oversimplified structural models and unacceptable inaccuracies on the estimates of the axial force, as it has been shown e.g. in $[9,24]$. The bending stiffness is usually treated as an additional unknown of the structural identification problem, due to the complex internal geometry of stay cables.

Boundary conditions are typically introduced in the form of either perfectly hinged or perfectly clamped cable end sections, to simplify the analytical treatment of the problem. A more realistic structural scheme could be defined, however, by considering equivalent translational and rotational springs at the beam end sections (see e.g. $[17,9]$ ) to model the flexibility of the restraint devices and of the support structures (e.g. deck and tower for cables in stayed bridges). Proper definition of equivalent springs strongly depends on the particular technology adopted to realize the restraints and is inherently related to several different sources of uncertainties, such as those related to geometric imperfections and aging of the support devices. Physical parameters characterizing the cable restraints, hence, should be added to the unknown of the structural identification problem.

Trying to circumvent this additional difficulty, identification procedures giving axial force estimates independent of the boundary conditions are recently surfacing in the literature $[10,59,60]$. They all rely on synchronous recording of the cable motion at several different locations along its length to get experimental information on the dynamic deflection shape of the element. The experimental setup and signal acquisition system, hence, turns out to be inherently more complex than the ones required by conventional identification techniques based on natural frequencies only. The latter, indeed, can be effectively implemented by acquiring acceleration signals at a single point of the cable. Standard dynamic testing techniques (see e.g. [49, 54]), then, can be used to get estimates of the lowest natural frequencies of the element.

In the present paper, we investigate the problem of the identification of cable axial force, on the basis of observed natural frequencies. Assuming a small relative bending stiffness of the cable elements, which is typical of stay cables and cement-grouted parallel-bundle wire cables, we provide an asymptotic expansion of the natural frequencies of cables with flexible supports in rotation and translation. This expansion is used to demonstrate that only two parameters can be identified in the asymptotic case of small bending stiffness. This model will be used to identify the axial force and the bending stiffness, while lumping all other problem parameters in a single dimensionless group.

The proposed model will then be exploited in order to formulate two different versions of an identification strategy: the first is based on a least-square approach embedded in a numerical procedure; the second relies on a standard linear regression in a conformal space. This latter approach is seen to generalize the current practice aiming a fitting the frequency vs. mode number relation obtained with simpler structural models. These two models are analyzed within the scope of an uncertainty quantification analysis. While the former provides accurate estimates of the confidence on the identified tension, the latter reveals by means of simple formulae the main quantities affecting the quality of the identification process.

\section{Transverse free-vibrations of stay cables}

\subsection{Statement of the problem}

Let us consider a stay cable of length $l$, with constant bending stiffness $E I$ and mass per unit of length $m$, subject to the axial force $T>0$. The cable is assumed to be anchored to flexible supports, herein modeled by means of uncoupled translational and rotational springs with stiffness coefficients $K_{T j} \geq 0$ and $K_{R j} \geq 0(j=0,1)$, as it is schematically depicted in Fig. 1 .

Stay cables are typically characterized by small values of both the sag-to-span ratio (in the order of few percents [6]) and the Irvine's parameter $\lambda^{2}$ (usually lower than one, with slightly larger values associated to very long stay cables [6]). As a consequence, both in-plane and out-of-plane small vibrations can be effectively described through the unstreatchable Euler-Bernoulli straight beam model. By neglecting damping, hence, planar transverse free vibrations are governed by the partial differential equation (e.g. [26]):

$$
E I \partial_{x}^{4} v-T \partial_{x}^{2} v+m \partial_{t}^{2} v=0
$$


where the function $v=v(x, t)$ describes the transverse displacements of the cable centerline, $x \in[0, l]$ is a coordinate spanning the chord of the element and $t \in \mathbb{R}^{+}$is the time.

The equation of motion (1) can be integrated, for prescribed initial conditions on the displacement and velocity fields $v(x, 0)$ and $\dot{v}(x, 0)$, under the boundary conditions:

$$
\left\{\begin{array}{l}
E I \partial_{x}^{3} v(0, t)-T \partial_{x} v(0, t)+K_{T 0} v(0, t)=0 \\
E I \partial_{x}^{3} v(l, t)-T \partial_{x} v(l, t)-K_{T 1} v(l, t)=0 \\
E I \partial_{x}^{2} v(0, t)-K_{R 0} \partial_{x} v(0, t)=0 \\
E I \partial_{x}^{2} v(l, t)+K_{R 1} \partial_{x} v(l, t)=0 .
\end{array}, \forall t>0\right.
$$

Stationary oscillatory solutions of Eq. (1) can be sought in the form:

$$
v(x, t)=\Phi(x) \sin (\Omega t+\Theta),
$$

where $\Omega$ is the vibration frequency, $\Theta$ is a constant phase depending on the initial conditions of the problem and $\Phi(x)$ is a mode shape. Substitution of Eq. (3) in Eqs. (1) and (2) yields the fourth order Sturm-Liouville problem defined by the ordinary differential equation:

$$
E I \Phi^{\prime \prime \prime \prime}-T \Phi^{\prime \prime}-\Omega^{2} \Phi=0, \Phi=\Phi(x), x \in[0, l]
$$

along with the boundary conditions:

$$
\left\{\begin{array}{l}
E I \Phi^{\prime \prime \prime}(0)-T \Phi^{\prime}(0)+K_{T 0} \Phi(0)=0 \\
E I \Phi^{\prime \prime \prime}(l)-\Phi^{\prime}(l)-K_{T 1} \Phi(l)=0 \\
E I \Phi^{\prime \prime}(0)-K_{R 0} \Phi^{\prime}(0)=0 \\
E I \Phi^{\prime \prime}(l)+K_{R 1} \Phi^{\prime}(l)=0
\end{array}\right.
$$

Please notice that in Eqs. (4) and (5) the apex denotes derivation with respect to $x$, i.e. $(\cdot)^{\prime}=\frac{d(\cdot)}{d x}$.

As it is well known, the problem (4)-(5) admits countably infinite non trivial solutions: $\left\{\Omega_{k}, \Phi_{k}(x)\right\}$, $k \in \mathbb{N}^{+}$. Exact closed form solutions can only be obtained in the particular case of doubly-hinged stay cables (e.g. [26]) and read:

$$
\begin{gathered}
\Omega_{k}=k \pi \sqrt{\frac{T}{m l^{2}}} \sqrt{1+(k \pi)^{2} \frac{E I}{T l^{2}}}, k \in \mathbb{N}^{+} \\
\Phi_{k}(x)=A_{k} \sin \left(\frac{\Omega_{k}}{V_{T}} x\right), k \in \mathbb{N}^{+}
\end{gathered}
$$

where $A_{k} \in \mathbb{R}$ is the modal displacement amplitude and $V_{T}=\sqrt{T / m}$ is the propagation speed of transverse waves in a taut string with mass per unit of length $m$ subject to the axial force $T$ (e.g. $[32,31])$.

For any other boundary conditions, such as those described by Eq. (5), exact closed form solutions of the boundary value problem (3)-(4) are not available and the modal properties (natural frequencies and mode shapes) of the structure are typically obtained through numerical solution strategies.

\subsection{Non-dimensional formulation}

The dynamic problem formulated in Section 2.1 can be restated in a non-dimensional form by introducing the characteristic frequency

$$
\Omega_{0}=\frac{V_{T}}{l}=\sqrt{\frac{T}{m l^{2}}},
$$




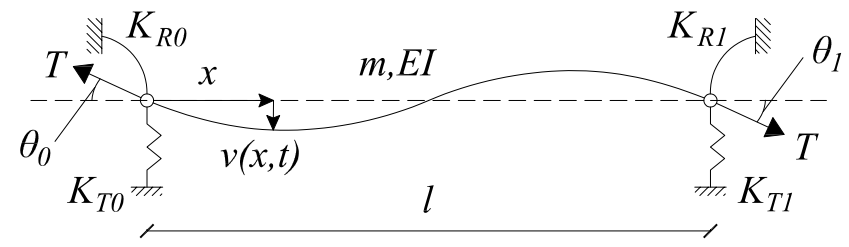

Figure 1: Schematic representation of a stay cable anchored to flexible supports and subject to a tensile load $T$. Cross sections are characterized by constant mass per unit of length $m$ and bending stiffness $E I$. The distance between the supports is denoted as $l$. Please notice that, under the small displacement assumption, at leading order the following equations hold true: $T \cos \left(\theta_{0}\right)=T \cos \left(\theta_{1}\right)=T$.

and the non-dimensional bending stiffness

$$
\varepsilon=\sqrt{\frac{E I}{T l^{2}}}
$$

Values of $\varepsilon$ typical of stay cables are lower than $0.01[6,7,45]$. Slightly higher values of $\varepsilon$, up to $\varepsilon=0.02-0.03$, can also be found in cement-grouted parallel-bundle wire cables used in stayed bridges [24,9] or in comparatively shorter cables used e.g. in tensile structures [8].

Substitution of Eqs. (8) and (9) in (1), yields the non-dimensional governing equation

$$
\varepsilon^{2} \partial_{\xi}^{4} \nu-\partial_{\xi}^{2} \nu+\partial_{\tau}^{2} \nu=0
$$

where $\xi=x / l \in[0,1]$ is the non-dimensional coordinate, $\tau=\omega_{0} t$ is the non-dimensional time and $\nu(\xi, \tau)=v(x(\xi), t(\tau)) / l$ is the non-dimensional transverse displacement of the cable centerline. The Eq. of motion (10) can be integrated, for prescribed initial conditions, under suitable boundary conditions that can be easily obtained from Eq. (2) and are herein omitted for the sake of conciseness.

Stationary oscillatory solutions of Eq. (10) can be expressed as $\nu(\xi, \tau)=\phi(\xi) \sin (\omega \tau+\Theta)$, where $\omega$ and $\phi(\xi)$ are the non-dimensional counterparts of the vibration frequency $\Omega$ and mode shape function $\Phi(x)$ in Eq. (3), i.e. $\omega=\Omega / \Omega_{0}$ and $\phi(\xi)=\Phi(x(\xi))$. After some straightforward computations, the boundary value problem (4)-(5) can be re-written as

$$
\begin{gathered}
\varepsilon^{2} \phi^{\prime \prime \prime \prime}-\phi^{\prime \prime}-\omega^{2} \phi=0, \quad \text { with } \xi \in[0,1] \\
\left\{\begin{array}{l}
\varepsilon^{2} \phi^{\prime \prime \prime}(0)-\phi^{\prime}(0)+k_{T 0} \phi(0)=0 \\
\varepsilon^{2} \phi^{\prime \prime \prime}(1)-\phi^{\prime}(1)-k_{T 1} \phi(1)=0 \\
\varepsilon^{2} \phi^{\prime \prime}(0)-k_{R 0} \phi^{\prime}(0)=0 \\
\varepsilon^{2} \phi^{\prime \prime}(1)+k_{R 1} \phi^{\prime}(1)=0
\end{array}\right.
\end{gathered}
$$

where the apex denotes derivation with respect to $\xi\left(\right.$ i.e. $\left.(\cdot)^{\prime}=\frac{d(\cdot)}{d \xi}\right)$, while $k_{T j}$ and $k_{R j}(j=0,1)$ are non-dimensional translational and rotational stiffness coefficients defined, respectively, as 

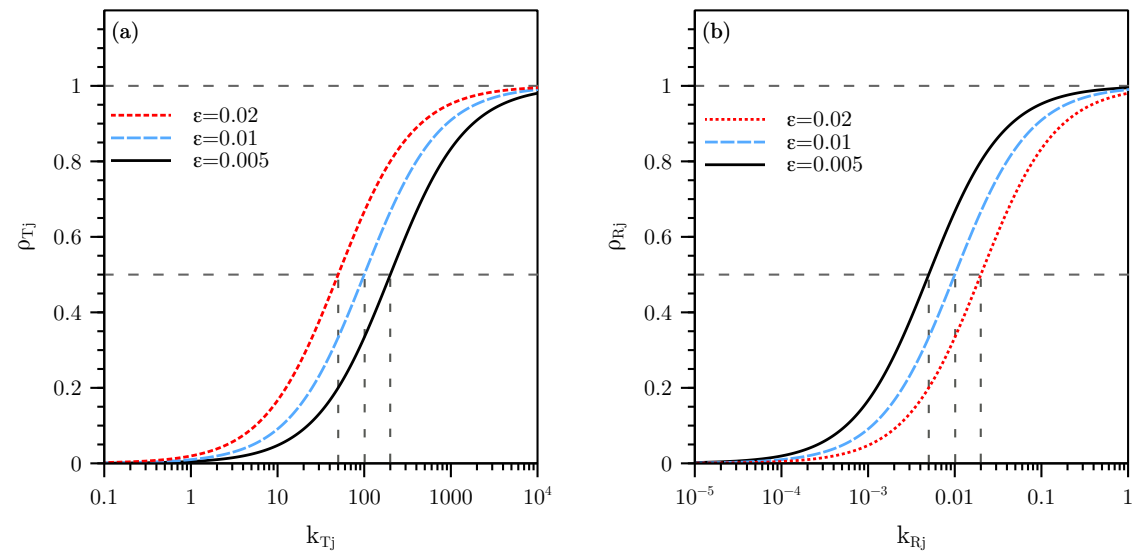

Figure 2: Translational ( $\rho_{T j}$, Fig. (a)) and rotational $\left(\rho_{R j}\right.$, Fig. (b)) degree-of-fixity parameters as a function of the corresponding non-dimensional stiffness coefficients. The results are shown for different values of the nondimensional bending stiffness $\varepsilon$.

$$
k_{T j}=\frac{l}{T} K_{T j} \quad \text { and } \quad k_{R j}=\frac{1}{T l} K_{R j}, \quad \text { with } j=0,1 .
$$

Both translational $\left(k_{T j}\right)$ and rotational $\left(k_{R j}\right)$ non-dimensional stiffness coefficients can take values on the left-bounded interval $I_{k}=[0,+\infty)$, with free and perfectly restrained boundary conditions corresponding, respectively, to the lower bound value $k_{-}=0$ and to the limit value $k_{+} \rightarrow \infty$. For modeling purposes, however, it is more convenient to characterize the translational and rotational cable restraints by means of degree-of-fixity parameters taking values in the closed unit interval $I_{\rho}=[0,1]$, with free and perfectly fixed restrained boundary conditions corresponding, respectively, to the lower and upper bound values $\rho_{-}=0$ and $\rho_{+}=1$ (e.g. $[9,33]$ ).

Starting from this observation, the following definitions are introduced in the present work for the translational $\left(\rho_{T j}\right)$ and rotational $\left(\rho_{R j}\right)$ degree-of-fixity parameters

$$
\begin{gathered}
\rho_{T j}=\frac{\varepsilon k_{T j}}{1+\varepsilon k_{T j}}, j=0,1 \\
\rho_{R j}=\frac{k_{R j}}{\varepsilon+k_{R j}}, j=0,1
\end{gathered}
$$

Equations (14) and (15) define two mappings of the left-bounded interval $I_{k}$ onto the closed unit interval $I_{\rho}$. Figures 2(a) and 2(b) respectively show the values of the translational and rotational degree-of-fixity parameters as a function of the corresponding non-dimensional stiffness coefficients for different values of the non-dimensional bending stiffness $\varepsilon$ typical of stay cables. It can be observed how the translational (rotational) degree-of-fixity parameter asymptotically approaches the unit value, corresponding to perfectly fixed boundary conditions, with increasing rapidity for increasing (decreasing) values of $\varepsilon$. The transition from 0 to 1 in $\rho_{R j}$ takes place for $k_{R j} \sim \varepsilon$ while the transition from 0 to 1 in $\rho_{T j}$ takes place for $k_{T j} \sim \varepsilon^{-1}$.

By exploiting the definitions in (14) and (15), then, the boundary conditions (12) can be re-written as

$$
\left\{\begin{array}{l}
\left(1-\rho_{T 0}\right)\left(\varepsilon^{3} \phi^{\prime \prime \prime}(0)-\varepsilon \phi^{\prime}(0)\right)+\rho_{T 0} \phi(0)=0 \\
\left(1-\rho_{T 1}\right)\left(\varepsilon^{3} \phi^{\prime \prime \prime}(1)-\varepsilon \phi^{\prime}(1)\right)-\rho_{T 1} \phi(1)=0 \\
\left(1-\rho_{R 0}\right) \varepsilon^{2} \phi^{\prime \prime}(0)-\rho_{R 0} \varepsilon \phi^{\prime}(0)=0 \\
\left(1-\rho_{R 1}\right) \varepsilon^{2} \phi^{\prime \prime}(1)+\rho_{R 1} \varepsilon \phi^{\prime}(1)=0
\end{array}\right.
$$


This formulation of the boundary conditions generalizes several interesting particular cases. Indeed, by setting $\rho_{T j}=\rho_{R j}=1$ in the above equation, the boundary conditions $\phi=0$ and $\varepsilon \phi^{\prime}=0$ at both ends is recovered. They correspond to the perfectly restrained problem in translation and rotation (provided $\varepsilon \neq 0$, the end rotation is equal to zero; otherwise the rotation cannot be specified). By setting $\rho_{T j}=1$ and $\rho_{R j}=0$, one recovers the boundary conditions of the hingedhinged stay cable while setting $\rho_{T j}=\rho_{R j}=0$ corresponds to the free-free stay cable.

As it has been previously mentioned, values of the non-dimensional bending stiffness typical of stay cables are much smaller than unity, i.e. $\varepsilon \ll 1$. The small number $\varepsilon$ multiplying the highest order derivative in Eq. (11) makes the boundary value problem (11)-(16) singularly perturbed and hints the existence of boundary layers in the mode shapes of the stay cable (e.g. $[55,27,19])$. It is worth noticing that the singular perturbation of Eq. (11) can also entail ill-conditioning, for vanishingly small values of $\varepsilon$, of numerical solvers associated to classic discretization techniques such as Finite Difference or Finite Element formulations (see e.g. [18]).

In the ideal limit case characterized by $\varepsilon=0$, the order of Eq. (11) is lowered from four to two. The resulting degenerate ordinary differential equation reads

$$
\phi^{\prime \prime}+\omega^{2} \phi=0, \phi=\phi(\xi), \quad \text { with } \xi \in[0,1]
$$

and the boundary conditions (16), i.e. $\phi(0)=\phi(1)=0$. It corresponds to the classic taut string model (e.g. $[32,31])$. The non-dimensional mode shapes are $\phi_{k}^{(t s)}(\xi)=a_{k} \sin \left(\omega_{k}^{(t s)} \xi\right)$, with $a_{k} \in \mathbb{R}$, and the dimensionless natural frequencies, obtained as integer multiples of the fundamental one, simply read

$$
\omega_{k}^{(t s)}=k \pi, \quad \text { with } k \in \mathbb{N}^{+}
$$

\subsection{Semi-analytical solution}

General solutions of Eq. (11) can be expressed as:

$$
\phi(\xi)=\alpha_{1} \sin \left(z_{1} \xi\right)+\alpha_{2} \cos \left(z_{1} \xi\right)+\alpha_{3} \exp \left(-z_{2} \xi\right)+\alpha_{4} \exp \left(-z_{2}(1-\xi)\right)
$$

where $\alpha_{i} \in \mathbb{R}(i=1, \ldots, 4)$ are integration constants, while the arguments $z_{1}$ and $z_{2}$ are the two functions of the non-dimensional vibration frequency $\omega$ :

$$
z_{j}=z_{j}(\omega)=\frac{1}{\varepsilon \sqrt{2}} \sqrt{(-1)^{j}+\sqrt{1+(2 \varepsilon \omega)^{2}}}, j=1,2 .
$$

Notice that using exponential instead of hyperbolic functions in Eq. (19) is more appropriate to highlight the existence of two boundary layers when $\varepsilon \ll 1$. Substitution of Eqs. (19) and (20) in the boundary conditions (16) yields the algebraic eigenvalue problem:

$$
\mathbf{B}(\omega ; \mathscr{P}) \boldsymbol{\alpha}=\mathbf{0}
$$

where $\boldsymbol{\alpha}=\left(\alpha_{1}, \ldots, \alpha_{4}\right)^{T}$ is a column vector listing the integration constants of Eq. (19), $\mathbf{0}$ is the four-dimensional null column vector and $\mathbf{B}$ is the $4 \times 4$ "boundary condition matrix" depending on $\omega$ and on the set of parameters: $\mathscr{P}=\left\{\varepsilon, \rho_{T 0}, \rho_{T 1}, \rho_{R 0}, \rho_{R 1}\right\}$. Closed form expressions for the components of the matrix $\mathbf{B}$ are fully reported in Appendix A. These, again, generalize simpler formulations known in particular cases.

Countably infinite non trivial solutions $\left\{\omega_{k}, \boldsymbol{\alpha}_{k}\right\}$, with $k \in \mathbb{N}^{+}$, of the algebraic eigenvalue problem (21) can be readily obtained by complementing Eq. (21) with the characteristic equation

$$
D(\omega ; \mathscr{P})=\operatorname{det}[\mathbf{B}(\omega ; \mathscr{P})]=0
$$

The eigenvalues $\omega_{k}$, i.e. the non-dimensional natural frequencies of the stay cable, correspond to the roots of Eq. (22). Generally speaking, hence, they can be regarded as functions of the five parameters belonging to the set $\mathscr{P}$. Intuitive symmetry reasons, however, allow one 
to observe that the eigenvalues $\omega_{k}$ should depend on two mappings $\rho_{T}$ and $\rho_{R}$ of the degree-offixity parameters that need to satisfy the symmetry conditions: $\rho_{T}\left(\rho_{T 0}, \rho_{T 1}\right)=\rho_{T}\left(\rho_{T 1}, \rho_{T 0}\right)$ and $\rho_{R}\left(\rho_{R 0}, \rho_{R 1}\right)=\rho_{R}\left(\rho_{R 1}, \rho_{R 0}\right)$. This indicates that the non-dimensional natural frequencies of the cable shall not depend on the 5 parameters of the problem, independently, but rather by means of some dimensionless groups.

For instance, symmetry requirements allowed to state that the non-dimensional natural frequencies of the cable should depend (at most) only on the non-dimensional bending stiffness $\varepsilon$ and on two parameters $\left(\rho_{T}\right.$ and $\left.\rho_{R}\right)$ describing the boundary conditions, i.e. $\omega_{k}=\omega_{k}\left(\varepsilon, \rho_{T}, \rho_{R}\right)$. Appropriate definitions for the parameters $\rho_{T}$ and $\rho_{R}$ will be introduced later in this Section. Notice that this discussion on symmetry is only valid for the natural frequencies but not for mode shapes.

Exact solutions of the characteristic Eq. (22) are only available for the special case of doublyhinged cables. They correspond to the non-dimensional counterparts of (6) and read

$$
\omega_{k}=k \pi \sqrt{1+(k \pi)^{2} \varepsilon^{2}} .
$$

For other boundary conditions, the roots of Eq. (22) can be numerically evaluated through a suitable root finding algorithm.

Beside numerical solutions, a deeper insight into the properties of the functions $\omega_{k}=\omega_{k}\left(\varepsilon, \rho_{T}, \rho_{R}\right)$ can be gained by expanding the determinant $D(\omega ; \mathscr{P})$ in Taylor series around $\varepsilon=0$. By truncating the series expansion at first order in $\varepsilon$ and focusing on the engineering meaningful case of non-zero translational stiffness of the anchorages (i.e. $\rho_{T 0}, \rho_{T 1}>0$ ), one can get

$$
D(\omega)=\rho_{T 0} \rho_{T 1}\left\{\sin (\omega)-2 \omega \cos (\omega)\left[1+\frac{\rho_{R 0}+\rho_{R 1}}{2}-\frac{1}{2}\left(\frac{1}{\rho_{T 0}}+\frac{1}{\rho_{T 1}}\right)\right] \varepsilon\right\}+o(\varepsilon)
$$

where $o($ ) denotes the Landau symbol ("little-o"). Inspection of Eq. (24) naturally leads to the following definitions for the parameters $\rho_{T}$ and $\rho_{R}$

$$
\rho_{R}=\frac{1}{2}\left(\rho_{R 0}+\rho_{R 1}\right) \quad \text { and } \quad \rho_{T}=\frac{2 \rho_{T 0} \rho_{T 1}}{\rho_{T 0}+\rho_{T 1}}
$$

Moreover, by introducing the parameter

$$
p=1+\rho_{R}-\frac{1}{\rho_{T}},
$$

Eq. (24) can be re-written in the more compact form

$$
D(\omega)=\rho_{T 0} \rho_{T 1}(\sin (\omega)-2 p \varepsilon \omega \cos (\omega))+o(\varepsilon) .
$$

The non-dimensional cable frequencies, hence, should satisfy the first order accurate transcendental equation

$$
\tan (\omega)=2 p \omega \varepsilon+o(\varepsilon)
$$

whose first-order accurate solutions read

$$
\omega_{k} \equiv \omega_{k}(\varepsilon, p)=k \pi(1+2 p \varepsilon)+o(\varepsilon), k \in \mathbb{N}^{+}
$$

Equations (28) and (29) clearly allow to appreciate how, for $\varepsilon \ll 1$, boundary conditions affect the lower-order non-dimensional cable frequencies through the global parameter $p$ only, rather than through the two independent parameters $\rho_{T}$ and $\rho_{R}$. This latter point, that will be further investigated in the next Section, has a major impact on the setting up of frequency-based axial force identification procedures, since it suggests that the progression of the natural frequencies of a stay cable anchored on flexible supports depends at first order only on two parameters, namely the non-dimensional bending stiffness $\varepsilon$ and the parameter $p$ defined in Eq. (26).

Moreover, Eq. (27) allows to conclude that, thanks to the scaling of the governing equations herein adopted, the determinant $D(\omega ; \mathscr{P})$ of the boundary condition matrix admits a Taylor series 
expansion with a bounded and order-one leading order term for vanishingly small values of $\varepsilon$. This makes searching for the roots of the characteristic Eq. (22) a well-conditioned problem that can be efficiently solved by means of simple root finding algorithms.

In the present work, the zeros of Eq. (22) are found through sequential applications of a standard dichotomy root finding algorithm. As an example, Fig. (3-a) shows the absolute value of the determinant $D(\omega)$ for a doubly-hinged stay cable $(p=0)$ and two different values of nondimensional bending stiffness, namely: $\varepsilon=0.01$ and $\varepsilon=0.02$. The red circles denote the first ten zeros of the function, numerically evaluated with a tolerance equal to $10^{-5}$ on $|D(\omega)|$. As it can be easily inferred from Fig. (3-b), for both values of $\varepsilon$ the non-dimensional frequencies obtained through the proposed semi-analytical solution strategy are in excellent agreement with the exact values calculated through Eq. (23). Fig. (3-b) also shows a comparison with the first ten natural frequencies of a Finite Element Model (FEM) relying on a discretization of the cable in 100 three-dimensional (3D) two-node equally spaced corotational Euler-Bernoulli beam elements [23, 22], fully accounting for the geometric nonlinearities that characterize the cable response and with consistent mass matrix. Focusing on planar vibration modes, displacements of the 3D finite elements have been constrained to belong to the vertical plane. As a consequence, each node of the elements can only undergo to transverse and longitudinal displacements and planar rotations (which amounts to a total number of 300 degrees-of-freedom, in the most general case of elastically restrained end sections). The beam elements are assumed to be straight in the reference configuration of the problem. Cubic and linear shape functions are adopted, respectively, for transverse and axial displacements. Preliminary parametric analyses have been performed by varying the number of $\mathrm{FE}$ to carefully check the convergence of the discrete model in terms of the lower natural frequencies and mode shapes. Preliminary comparisons with the outcomes of a lumped mass FEM have also have been carried out, leading as expected (due to the inherent slenderness of the structural element herein considered) to practically negligible differences in the results. The natural frequencies of the FEM are in good agreement with both the semi-analytical and the exact solutions, with maximum discrepancies smaller than $1 \%$ for the tenth mode.

The validity of the proposed semi-analytical model has been further assessed, under different boundary conditions, through extensive comparisons with the outcomes of the FEM. Results are shown in Figure 4 in terms of the first ten non-dimensional natural frequencies of stay cables with non-dimensional bending stiffness equal to $\varepsilon=0.01$ (Fig. 4(a)) and $\varepsilon=0.02$ (Fig. 4(b)) and the following five different boundary conditions: (I) $\rho_{R}=0, \rho_{T}=1(p=0$, doubly-hinged stay cable), (II) $\rho_{R}=1, \rho_{T}=1\left(p=1\right.$, doubly-clamped stay cable), (III) $\rho_{R}=0.5, \rho_{T}=1(p=0.5)$, (IV) $\rho_{R}=1, \rho_{T}=0.5(p=0),(\mathrm{V}) \rho_{R}=1, \rho_{T}=0.25(p=-2)$. It can be observed that the outcomes of the two models are in excellent agreement for all values of $\varepsilon$ and boundary conditions herein considered, with maximum discrepancies smaller than $1 \%$ for the tenth mode.

It is also interesting to notice that the curves corresponding to the boundary conditions (I) and (IV), associated to the same value of restraint parameter $p=0$, are practically coincident up to the fifth and third mode for respectively $\varepsilon=0.01$ and $\varepsilon=0.02$. Differences between the curves increase with the order of the mode, but are less than $0.5 \%$ and $4 \%$ at the tenth mode for respectively $\varepsilon=0.01$ and $\varepsilon=0.02$. These numerical results further strengthen the conclusion, previously reached through inspection of Eqs. (28) and (29), that lower-order non-dimensional frequencies of stay cables are affected by the global restraint parameter $p$ only, rather than by the two independent parameters $\rho_{T}$ and $\rho_{R}$.

\subsection{Closed-form asymptotic solution}

Closed form equations for the natural frequencies of stay cables are of valuable interest to define efficient vibration-based axial force identification procedures. As it has been already mentioned in Section 2.3, however, exact closed form solutions of the fourth order Sturm-Liouville problem (11)-(16) can only be found in the special case of doubly-hinged cables (see Eq. (23)). During 

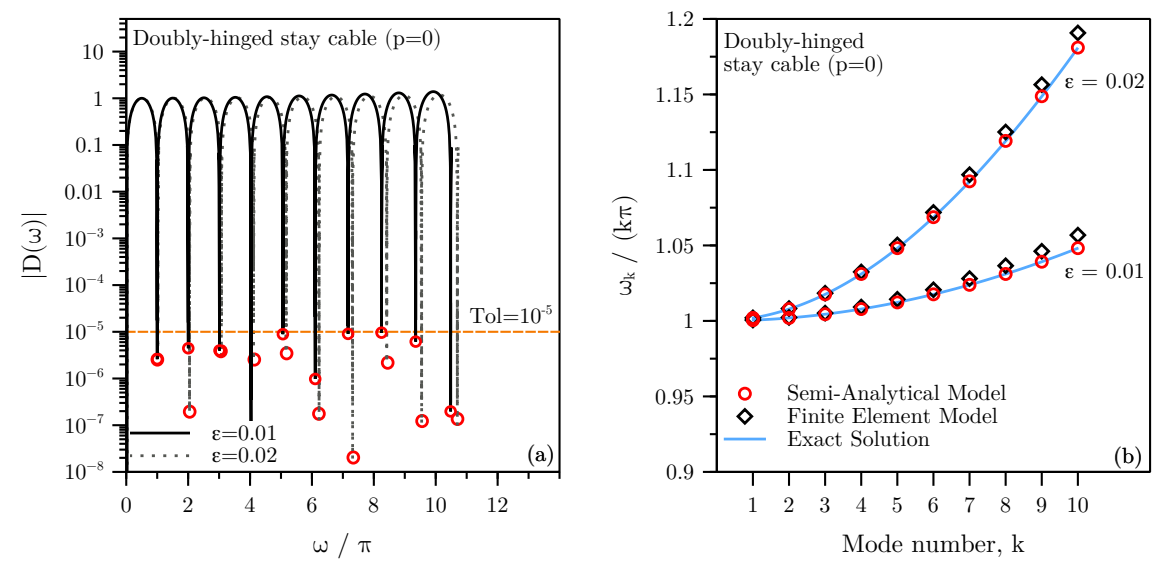

Figure 3: Doubly-hinged stay cable $(p=0)$. Results are shown for two different values of the non-dimensional bending stiffness $\varepsilon$. (a) Absolute value of the determinant $D(\omega)$ of the boundary condition matrix (Eq. (22)). The red circles denote the zeros of the determinant, numerically evaluated with a tolerance equal to $10^{-5}$ on $|D(\omega)|$. (b) Non-dimensional natural frequencies: comparison among the results of the proposed semi-analytical model, the exact solution (Eq. (23)) and the outcomes of a finite element model.
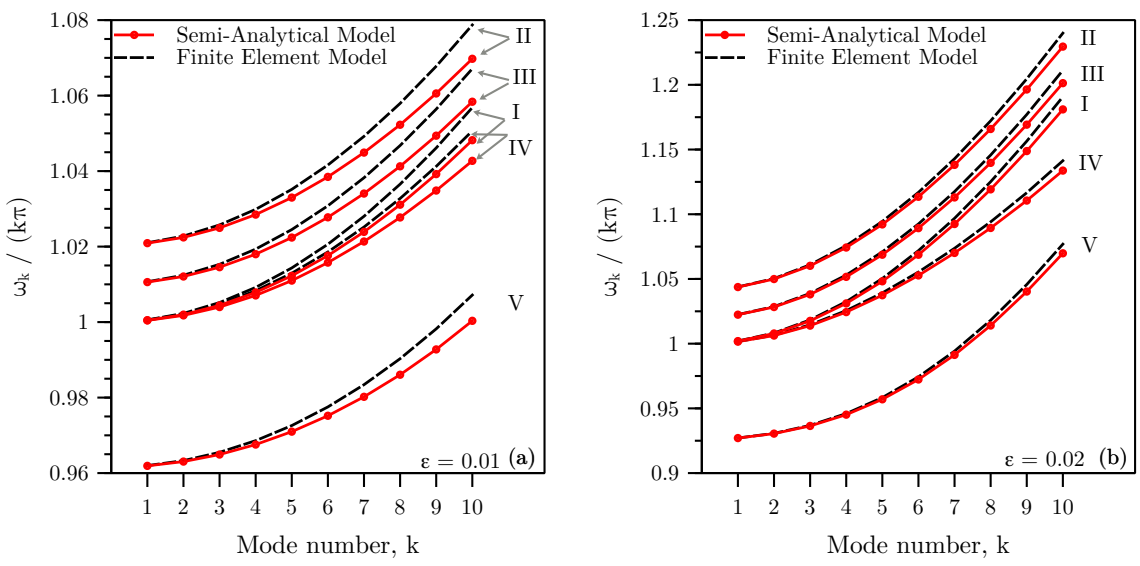

Figure 4: Comparison between the non-dimensional natural frequencies obtained through the semi-analytical and the finite element model. Results are shown for $\varepsilon=0.01$ (Fig. (a)) and $\varepsilon=0.02$ (Fig. (b)) and five different boundary conditions: (I) $\rho_{R}=0, \rho_{T}=1\left(p=0\right.$, doubly-hinged stay cable), (II) $\rho_{R}=1, \rho_{T}=1(p=1$, doublyclamped stay cable), (III) $\rho_{R}=0.5, \rho_{T}=1(p=0.5),(\mathrm{IV}) \rho_{R}=1, \rho_{T}=0.5(p=0),(\mathrm{V}) \rho_{R}=1, \rho_{T}=0.25$ $(p=-2)$. 
the years, this observation led researchers to adopt both pragmatical approaches and approximate solution strategies.

Pragmatical approaches of the literature mainly consist in: (a) neglecting the effects of the bending stiffness, (e.g. [12, 25, 4]) so that Eqs. (11)-(16) boil down to the taut string eigenvalue problem (17), whose natural frequencies are reported in Eq. (18); or (b) properly accounting for the effects of the bending stiffness, but approximately modeling cable anchorages by assuming a doubly-hinged structural scheme (e.g. [2]). It is worth noting that, for values of the nondimensional bending stiffness $\varepsilon$ typical of stay cables, both pragmatical approaches (a) and (b) usually deliver an excellent estimate of the fundamental frequency, while higher order natural frequencies are predicted with a level of accuracy decreasing with the modal order.

Approximate closed form expressions of the stay cable natural frequencies have also been obtained in the literature under particular assumptions on the boundary conditions, namely for the doubly-clamped and the doubly-hinged structural schemes. Focusing on doubly-clamped axiallyloaded elements, Morse and Ingard [46] derived, through a Taylor series expansion of the terms of the characteristic equation (22), a second-order accurate asymptotic expression later used for axial force identification purposes by several authors (e.g. $[17,24,6]$ )

$$
\omega_{k}=k \pi\left(1+2 \varepsilon+\left(4+\frac{(k \pi)^{2}}{2}\right) \varepsilon^{2}\right)+o\left(\varepsilon^{2}\right), k \in \mathbb{N}^{+}
$$

where the leading order term gives, as expected, the non-dimensional natural frequencies of the taut string model (cf. Eq. (18)). By following the same approach as Morse and Ingard [46], a similar result can also be easily obtained for doubly-hinged elements

$$
\omega_{k}=k \pi\left(1+\frac{(k \pi)^{2}}{2} \varepsilon^{2}\right)+o\left(\varepsilon^{2}\right), k \in \mathbb{N}^{+}
$$

Notice that Eq. (31) can also be readily obtained through a Taylor series expansion of the exact solution (23).

A novel second-order accurate closed form asymptotic expression, that generalizes Eqs. (30) and (31) to account for a partial flexibility of cable anchorages, is developed in the following through a standard perturbation approach (e.g. [27]).

Let us search for a second-order accurate asymptotic solution of the algebraic eigenvalue problem (21) that can be expressed through the regular expansion

$$
\begin{gathered}
\omega=\omega_{(0)}+\omega_{(1)} \varepsilon+\omega_{(2)} \varepsilon^{2}+o\left(\varepsilon^{2}\right), \\
\boldsymbol{\alpha}=\boldsymbol{\alpha}_{(0)}+\boldsymbol{\alpha}_{(1)} \varepsilon+\boldsymbol{\alpha}_{(2)} \varepsilon^{2}+o\left(\varepsilon^{2}\right) .
\end{gathered}
$$

Substitution of Eq. (32) in the definitions (A.1)-(A.16) and subsequent Taylor series expansion in a neighborhood of $\varepsilon=0$, yields the following second-order accurate expression for the boundary condition matrix $\mathbf{B}$

$$
\mathbf{B}=\mathbf{B}_{(0)}\left(\omega_{(0)}\right)+\mathbf{B}_{(1)}\left(\omega_{(1)}, \omega_{(0)}\right) \varepsilon+\mathbf{B}_{(2)}\left(\omega_{(2)}, \omega_{(1)}, \omega_{(0)}\right) \varepsilon^{2}+o\left(\varepsilon^{2}\right)
$$

where $\mathbf{B}_{(0)}, \mathbf{B}_{(1)}$ and $\mathbf{B}_{(2)}$ are $4 \times 4$ matrices whose components are fully reported in Appendix B. Substitution of Eqs. (33) and (34) in (21), then, leads to the matrix equation

$$
\left(\mathbf{B}_{(0)}+\mathbf{B}_{(1)} \varepsilon+\mathbf{B}_{(2)} \varepsilon^{2}\right)\left(\boldsymbol{\alpha}_{(0)}+\boldsymbol{\alpha}_{(1)} \varepsilon+\boldsymbol{\alpha}_{(2)} \varepsilon^{2}\right)=\mathbf{0}
$$

The individual vanishing of the coefficients of the different powers in $\varepsilon$ in Eq. (35) yields the system of equations

$$
\begin{cases}\operatorname{ord}\left(\varepsilon^{0}\right): & \mathbf{B}_{(0)} \boldsymbol{\alpha}_{(0)}=\mathbf{0} \\ \operatorname{ord}\left(\varepsilon^{1}\right): & \mathbf{B}_{(0)} \boldsymbol{\alpha}_{(1)}+\mathbf{B}_{(1)} \boldsymbol{\alpha}_{(0)}=\mathbf{0} \\ \operatorname{ord}\left(\varepsilon^{2}\right): & \mathbf{B}_{(0)} \boldsymbol{\alpha}_{(2)}+\mathbf{B}_{(1)} \boldsymbol{\alpha}_{(1)}+\mathbf{B}_{(2)} \boldsymbol{\alpha}_{(0)}=\mathbf{0}\end{cases}
$$


that can be easily solved for the variables $\omega_{(i)}$ and $\boldsymbol{\alpha}_{(i)}(i=0,1,2)$ through a cascaded approach, starting from the leading order problem (i.e. $\operatorname{ord}\left(\varepsilon^{0}\right)$ ) and moving towards the higher order ones. Solution of (36) is fully detailed in Appendix C and leads to the following second-order accurate asymptotic expression for the non-dimensional natural frequencies of the cable

$$
\omega_{k}=k \pi\left(1+2 p \varepsilon+\left(\frac{(k \pi)^{2}}{2}+4 p^{2}\right) \varepsilon^{2}\right)+o\left(\varepsilon^{2}\right), k \in \mathbb{N}^{+}
$$

The leading order term in Eq. (37) coincides, as expected, with the frequencies $\omega_{k}^{(t s)}$ of the taut sting model (Eq. (18)), while the effects of the bending stiffness and of the flexibility of the cable anchorages enter the first and second order correction terms through the non-dimensional variables $\varepsilon$ and $p$. Equation (37), hence, can also be re-written in the more expressive form

$$
\omega_{k}=\omega_{k}^{(t s)}\left(1+f_{k}(\varepsilon, p)\right)+o\left(\varepsilon^{2}\right), k \in \mathbb{N}^{+}
$$

with the definition

$$
f_{k}(\varepsilon, p)=2 p \varepsilon+\left(\frac{(k \pi)^{2}}{2}+4 p^{2}\right) \varepsilon^{2}, k \in \mathbb{N}^{+}
$$

It is also worth noting that the proposed asymptotic solution easily allows one to recover the equations previously introduced for the special cases of doubly-clamped (Eq. (30)) and doublyhinged (Eq. (31)) cables, by respectively setting $p=1$ or $p=0$ in Eq. (39). More importantly, Equations (38-39) show that, at leading order the natural frequencies are given by the taut string model, $\omega_{k} \sim \omega_{k}^{(t s)}$. Then, the second and third order terms explicitly given in $f_{k}(\varepsilon, p)$ indicate that natural frequencies only depend on $p$ and $\varepsilon$. The only possibility to separate, on the sole basis of natural frequencies, the influence of translational and rotational flexibilities of anchorages, would be to push the derivation to the fourth order. For practical reasons, the measurement noise and epistemic uncertainties make it unrealistic to derive an identification procedure based on a fourth-order small detail. This also justifies the reason why the two identification procedures presented in the following Section (Sections 3.2 and 3.3) do not pretend to identify more than 3 parameters. Furthermore, Equations (38-39) indicate that the sequence of $\left\{\omega_{k}\right\}$, as a function of $k$, takes the form of a quadratic expression in $k$ with only two terms, namely the intercept and the second degree coefficient). The adjustement of a mathematical model to the sequence of $\left\{\omega_{k}\right\}$, as a function of $k$, is therefore not able to capture more than 2 independent parameters. This explains why $p$ is considered as a parameter, while the proposed identification techniques will provide estimators for the cable tension and bending stiffness.

Figure 5 shows a comparison between the outcomes of the asymptotic solution (38) and the ones of the semi-analytical model described in Section 2.3. Results are presented in terms of the first ten non-dimensional natural frequencies of stay cables with non-dimensional bending stiffness equal to $\varepsilon=0.01$ (Fig. 5(a)) and $\varepsilon=0.02$ (Fig. 5(b)) under the same five different boundary conditions labeled as (I)-(V) in Section 2.3. Notice that the asymptotic solutions corresponding to cases (I) and (IV) are coincident, since they are both associated to the same value of the non-dimensional restraint parameter: $p=0$.

On the overall, the asymptotic solution is in excellent agreement with the results of the semianalytical model. Discrepancies increase, as expected, with the increase of both $\varepsilon$ and the modal order. For $\varepsilon=0.01$ (Fig. 5(a)), differences are less than $1 \%$ over the whole range of modes herein considered and for all different boundary conditions. For $\varepsilon=0.02$ (Fig. 5(a)), the maximum differences between the asymptotic solution and the outcomes of the semi-analytical model are less than $1.5 \%$ for boundary conditions (I), (II) and (III). Discrepancies are larger for the boundary conditions (IV) and (V), being however in the order of $5 \%$ for the tenth mode. 

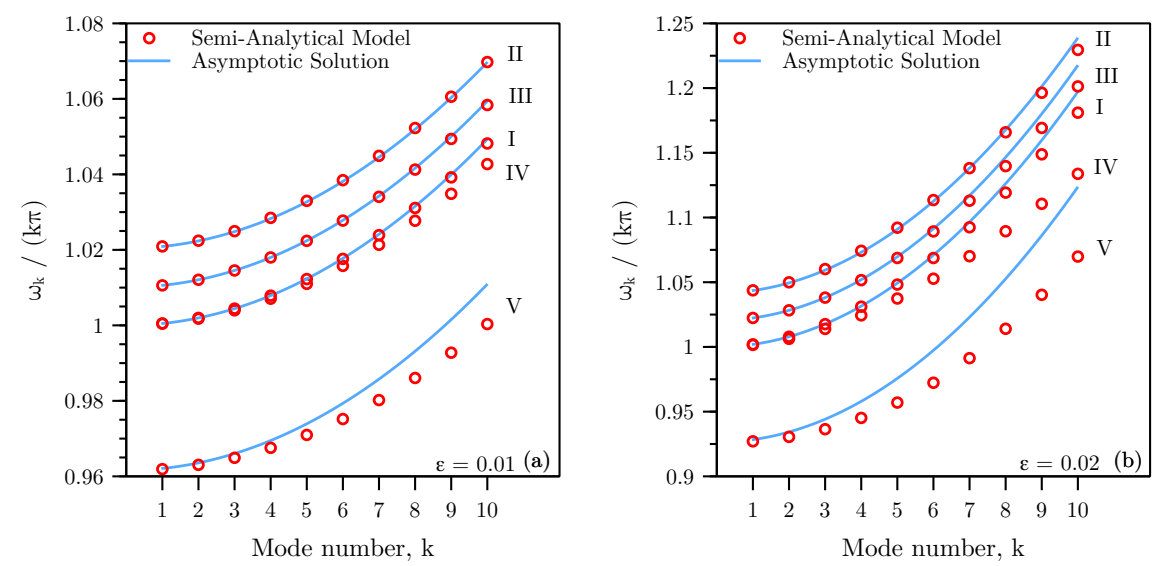

Figure 5: Comparison between the non-dimensional natural frequencies obtained through the asymptotic solution (Eq. (37)) and the semi-analytical model (Section 2.3). Results are shown for $\varepsilon=0.01$ (Fig. (a)) and $\varepsilon=0.02$ (Fig. (b)) and five different boundary conditions: (I) $\rho_{R}=0, \rho_{T}=1\left(p=0\right.$, doubly-hinged stay cable), (II) $\rho_{R}=1$, $\rho_{T}=1\left(p=1\right.$, doubly-clamped stay cable), (III) $\rho_{R}=0.5, \rho_{T}=1(p=0.5),(\mathrm{IV}) \rho_{R}=1, \rho_{T}=0.5(p=0),(\mathrm{V})$ $\rho_{R}=1, \rho_{T}=0.25(p=-2)$.

\section{Parameter identification problem}

Most vibration-based methods for the identification and monitoring of the axial force of stay cables rely on the knowledge of a set of experimentally determined natural frequencies. Stay cables are indeed lightweight and lightly damped structural elements, whose transverse vibrations can be easily excited by providing relatively small amounts of input energy. Standard dynamic testing techniques (see e.g. $[49,54]$ ), hence, can be effectively used to get estimates of the lowest natural frequencies of the cable. This experimental information, along with a suitable structural model, serves as the basis to set up a model updating strategy to identify the value of unknown structural parameters.

More specifically, assuming that the length $l$ and mass per unit length $m$ of the stay cable are known, the axial force in the stay cable is determined from (8):

$$
T=m l^{2} \Omega_{0}^{2},
$$

which indicates that the identification problem is limited to estimating $\Omega_{0}$.

Three different identification strategies are presented in this Section. Classic approaches relying on application of the taut string model are firstly reviewed in Section 3.1. The closed form asymptotic solution developed in Section 2.4 is used, within this context, to provide a rigorous assessment of the effects on the axial force estimate due to the simplifying assumptions at the base of the taut string model. Two novel strategies for the simultaneous identification of the axial force and bending stiffness of stay cables anchored to flexible supports are then presented in Sections 3.2 and 3.3. The first one (Section 3.2) relies on the numerical solution of a non-linear optimization problem, while the second one (Section 3.3) is based on the application of a simple linear regression model.

\subsection{Taut string model}

Let us denote as $\mathcal{M}^{*}=\left\{\Omega_{k_{1}}^{*}, \Omega_{k_{2}}^{*}, \ldots, \Omega_{k_{M}}^{*}\right\}$ a set of $M$ natural circular frequencies, identified from a vibration test and associated to the modes $k_{1} \leq k_{2} \leq \ldots \leq k_{M}$ (with $k_{j} \in \mathbb{N}^{+}, \forall j \in[1, M]$ ). The measured frequencies $\mathcal{M}^{*}$ can be conveniently regarded as realizations of a set of independent Gaussian variables $\mathcal{M}=\left\{W_{k_{1}}, W_{k_{2}}, \ldots, W_{k_{M}}\right\}$, with nominal average values and variances given by

$$
\bar{\Omega}_{k_{j}}=\mathbb{E}\left[W_{k_{j}}\right] \quad \text { and } \quad \sigma_{k_{j}}^{2}=\mathbb{E}\left[W_{k_{j}}^{2}\right]-\bar{\Omega}_{k_{j}}^{2}, \quad j=1, \ldots, M
$$


where $\mathbb{E}[$.$] denotes the mathematical expectation [48].$

The most simple axial force identification strategy proposed in the literature relies on the adoption of the taut string model $[17,7]$, whose natural circular frequencies $\Omega_{k}^{(t s)}$ are given by

$$
\Omega_{k}^{(t s)}=\Omega_{0} \omega_{k}^{(t s)}=k \pi \Omega_{0}, \quad k=1,2, \ldots
$$

since $\omega_{k}^{(t s)}=k \pi$ (see Eq. (18)). Due to both unavoidable measurements errors and the simplifying assumptions of the adopted structural model ("modeling errors"), the measured frequencies $\mathcal{M}^{*}$ will only approximately satisfy Eq. (42). An estimate $\hat{\Omega}_{0}$ of the characteristic frequency can be obtained by firstly solving for $\Omega_{0}$ each of the equations: $\Omega_{k_{j}}^{(t s)}=\Omega_{k_{j}}^{*}(j=1, \ldots, M)$, and then taking the arithmetic mean of the results, i.e.

$$
\hat{\Omega}_{0}=\frac{1}{M} \sum_{j=1}^{M} \frac{\Omega_{k_{j}}^{*}}{k_{j} \pi}, \quad M \geq 1 .
$$

Once $\hat{\Omega}_{0}$ is known from Eq. (43), the axial force can be estimated from Eq. (40) as

$$
\hat{T}=m l^{2} \hat{\Omega}_{0}^{2}
$$

where $m$ and $l$ should be regarded as the nominal values of the linear density and length of the cable. A simple and usual approach consists in considering only one natural frequency, in which case the summation drops and makes the determination of the estimate $\hat{\Omega}_{0}$ straightforward.

The bending stiffness of the stay cable and the flexibility of the anchorages, which are not accounted for in the taut string model, unfortunately make $\hat{\Omega}_{0}$ a biased estimator. The bias associated to these modeling errors can be quantitatively assessed by exploiting the asymptotic solution introduced in Section 2.4. Taking expectations of both sides of Eq. (43) yields

$$
\mathbb{E}\left[\hat{\Omega}_{0}\right]=\frac{1}{M} \sum_{j=1}^{M} \frac{\bar{\Omega}_{k_{j}}}{k_{j} \pi} .
$$

For small values of the non-dimensional bending stiffness $\varepsilon$ and focusing on the lower modes of the cable, the average values $\bar{\Omega}_{k_{j}}$ can be approximately assumed equal to $\bar{\Omega}_{k_{j}}=\Omega_{0} \omega_{k_{j}}^{(t s)}\left(1+f_{k_{j}}(\varepsilon, p)\right)$ (see Eq. (38)). Substitution in Eq. (45) yields

$$
\mathbb{E}\left[\hat{\Omega}_{0}\right]=\Omega_{0}\left(1+\frac{1}{M} \sum_{j=1}^{M} f_{k_{j}}(\varepsilon, p)\right)
$$

By recalling the definition of $f_{k}(\varepsilon, p)$ introduced in Eq. (39), the bias term in (46) reads

$$
\operatorname{bias}\left[\hat{\Omega}_{0}\right]=\mathbb{E}\left[\hat{\Omega}_{0}\right]-\Omega_{0}=\Omega_{0}\left(2 p \varepsilon(1+2 p \varepsilon)+\varepsilon^{2} \frac{\pi^{2}}{2 M} \sum_{j=1}^{M} k_{j}^{2}\right)
$$

Figure 6 shows this theoretical bias of the estimator $\hat{\Omega}_{0}$ as a function of the non-dimensional bending stiffness $\varepsilon$ and for three different boundary conditions: (I) $p=0$ (doubly-hinged stay cable), (II) $p=1$, (doubly-clamped stay cable) and (III) $p=0.5$. These boundary conditions cover the whole range of values that the restraint parameter $p$ can assume in the special case, often encountered in practice, of stay cables anchored to supports characterized by negligible translational flexibility (i.e. $\rho_{T}=1$ in Eq. (26)). Figures 6(a) and 6(b) are referred to identification procedures respectively based on the knowledge of (a) the fundamental frequency of the cable only (i.e. $j=M=1$ in Eqs. (45) and (47)), and (b) the five lower natural frequencies of the cable (i.e. $M=5$ and $j=1,2, \ldots, 5$ in Eqs. (45) and (47)).

As it can be easily observed from Figure 6 , the bias tends to zero for structural elements approaching the idealized taut string model, i.e. for vanishingly small values of $\varepsilon$. Moreover, the 

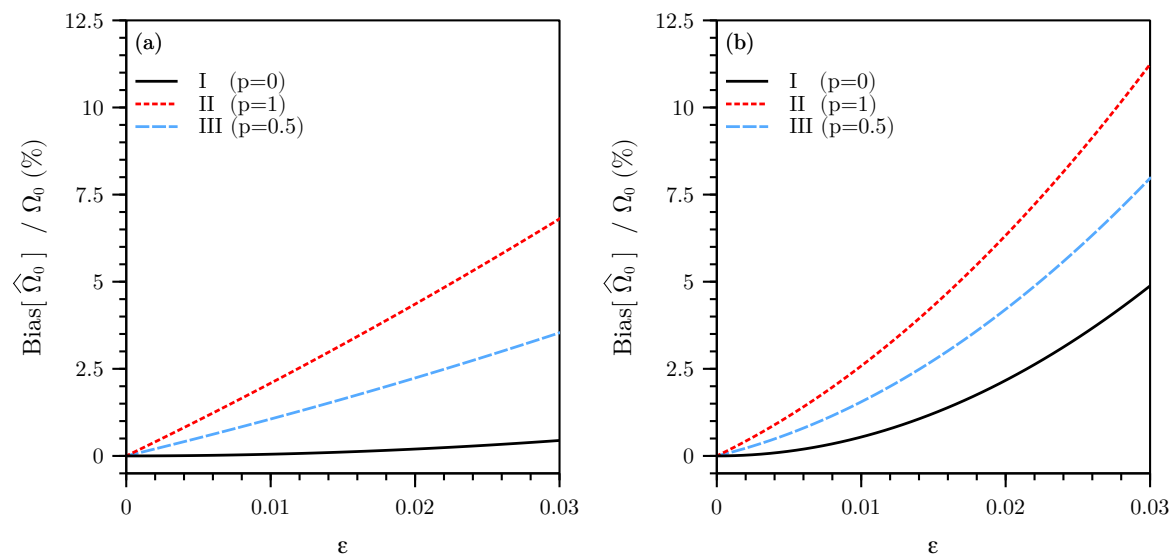

Figure 6: Bias of the estimator $\hat{\Omega}_{0}$ relying on the application of a taut string model (see Eqs. (43) and (47)). The bias is normalized for the characteristic frequency $\Omega_{0}$ and plotted as a function of the non-dimensional bending stiffness $\varepsilon$. Results are shown for three different boundary conditions: (I) $p=0$ (doubly-hinged stay cable), (II) $p=1$, (doubly-clamped stay cable) and (III) $p=0.5$. Figure (a) is obtained by considering $M=1$ and $j=1$ (fundamental mode of the cable). Figure (b) is obtained by considering $M=5$ and $j=1,2, \ldots, 5$ (first five modes of the cable).

bias increases with the increase of both (i) the number of modes $M$ considered in the identification procedure, and (ii) the value of the degree-of-fixity parameter $p$. For fixed values of $\varepsilon$ and $M$, indeed, the bias is minimum for doubly hinged stay cables $(p=0$, curves labeled as (I) in Fig. 6) and maximum for doubly clamped stay cables ( $p=1$, curves labeled as (II) in Fig. 6). It can be concluded, hence, that the bias of the estimator $\hat{\Omega}_{0}$ increases with the increase of the rotational stiffness of the cable anchorages.

On the overall, inspection of the results depicted in Figure 6, shows that, depending on the boundary conditions and the number of modes $M$, the bias introduced by modeling errors in the identification procedure based on the taut string model can be quite relevant, i.e. in the order of several percent of $\Omega_{0}$.

In spite of these potentially significant inaccuracies, identification strategies based on the taut string model are widely used in practice due to their inherent simplicity, paving the way for a straightforward assessment of uncertainties. With the probabilistic model (41) for the measured frequencies, the variance $\sigma_{\hat{\Omega}_{0}}^{2}$ of the estimator $\hat{\Omega}_{0}$ can be calculated as

$$
\sigma_{\hat{\Omega}_{0}}^{2}=\mathbb{E}\left[\hat{\Omega}_{0}^{2}\right]-\left(\mathbb{E}\left[\hat{\Omega}_{0}\right]\right)^{2}=\frac{1}{M^{2}} \sum_{j=1}^{M} \frac{\sigma_{k_{j}}^{2}}{k_{j}^{2} \pi^{2}}
$$

By assuming that the measured frequencies are characterized by a constant coefficient of variation $I_{n}=\sigma_{k_{n}} / \bar{\Omega}_{k_{n}}$ (also termed in the following, with a slight abuse of terminology, noise intensity), Eq. (48) can be re-written as

$$
\sigma_{\hat{\Omega}_{0}}^{2}=\frac{I_{n}^{2}}{M^{2}} \sum_{j=1}^{M} \frac{\bar{\Omega}_{k_{j}}^{2}}{k_{j}^{2} \pi^{2}} .
$$

The substitution of $\bar{\Omega}_{k_{j}}=\Omega_{0} \omega_{k_{j}}^{(t s)}\left(1+f_{k_{j}}(\varepsilon, p)\right)$ in Eq. (49), then, yields the following expression for the standard deviation $\sigma_{\hat{\Omega}_{0}}$

$$
\sigma_{\hat{\Omega}_{0}}=\frac{I_{n} \Omega_{0}}{M} \sqrt{\sum_{j=1}^{M}\left(1+f_{k_{j}}(\varepsilon, p)\right)^{2}}
$$

By neglecting higher-order terms, hence, $\sigma_{\hat{\Omega}_{0}}$ can be approximately computed as 


$$
\sigma_{\hat{\Omega}_{0}}=\frac{I_{n}}{\sqrt{M}} \Omega_{0} \simeq \frac{I_{n}}{\sqrt{M}} \hat{\Omega}_{0} .
$$

It is worth noticing that the standard deviation of the estimator $\hat{\Omega}_{0}$, contrarily to the bias due to modeling errors, decreases with the increase of the number of modes $M$. As a consequence, the selection of an appropriate value of $M$ naturally claims for a trade-off between accuracy and variability of the outcomes of the identification procedure.

Starting from Eq. (44) the combined uncertainty $\sigma_{\hat{T}}$ on the estimated value $\hat{T}$ of the cable axial force can be evaluated through the well-known first-order accurate expression (e.g. [17, 7]):

$$
\frac{\sigma_{\hat{T}}}{\hat{T}} \simeq \frac{\sigma_{m}}{m}+2 \frac{\sigma_{l}}{l}+2 \frac{\sigma_{\hat{\Omega}_{0}}}{\hat{\Omega}_{0}}
$$

where $\sigma_{m}$ and $\sigma_{l}$ are the standard uncertainties (see e.g. [5]) associated to the nominal values $m$ and $l$ of the cable linear density and length.

\subsection{Non-linear optimization problem}

Both bending stiffness and anchorage flexibility effects on the low-order natural circular frequencies $\Omega_{k}(k=1,2, \ldots)$ of a stay cable can be accurately accounted for by means of the closedform asymptotic solution presented in Section 2.4. Multiplication of Eq. (38) by the characteristic frequency $\Omega_{0}=\Omega_{0}(T, m, l)$ (see Eq.(8)) yields

$$
\Omega_{k}=\Omega_{0} \omega_{k}^{(t s)}\left(1+f_{k}(\varepsilon, p)\right), \quad k=1,2, \ldots
$$

where $\omega_{k}^{(t s)}=k \pi$ are the non-dimensional frequencies of the taut string model (Eq. (18)).

Whenever a set of $M$ measured frequencies $\mathcal{M}^{*}=\left\{\Omega_{k_{1}}^{*}, \Omega_{k_{2}}^{*}, \ldots, \Omega_{k_{M}}^{*}\right\}$ is available from vibration tests, the unknown model parameters $\Omega_{0}, \varepsilon$ and $p$ can be estimated by minimizing the difference between predictions of Eq. (53) and experimental observations. To this aim, let us introduce the cost (or objective) function

$$
F_{\text {obj }}(\boldsymbol{X})=\sqrt{\sum_{j=1}^{M}\left(1-\frac{\Omega_{k_{j}}}{\Omega_{k_{j}}^{*}}\right)^{2}}
$$

where $\boldsymbol{X}$ is the parameter vector $\boldsymbol{X}=\left(\Omega_{0}, \varepsilon, p\right)^{T} \in \mathcal{S} \subset \mathbb{R}^{3}$, taking values on the searching space $\mathcal{S}$ subject to the physical constraints: $\Omega_{0}>0, \varepsilon>0$ and $p \leq 1$. Whenever the translational flexibility of the cable anchorages can be assumed as negligible, the degree-of-fixity parameter $p$ can only take values in the closed unit interval, i.e. $0 \leq p \leq 1$ and the definition of the searching space $\mathcal{S}$ needs to be modified accordingly.

Identification of the structural parameters, within this context, amounts to solve the non-linear constrained optimization problem

$$
\hat{\boldsymbol{X}}=\underset{\boldsymbol{X} \in \mathcal{S}}{\operatorname{argmin}} F_{\text {obj }}(\boldsymbol{X})
$$

Once the optimal parameters $\hat{\Omega}_{0}$ and $\hat{\varepsilon}$ are known from the solution $\hat{\boldsymbol{X}}$ of (55), estimates of the cable axial force $(\hat{T})$ and bending stiffness $(\hat{E} I)$ can be respectively obtained through Eq. (44) and Eq. (9) as:

$$
E I=\hat{T} \hat{\varepsilon}^{2} l^{2}
$$

The non-linear optimization problem defined in Eq. (55) is characterized by several peculiar features that should guide the selection of an appropriate solution algorithm. Due to unavoidable measurement errors affecting the natural frequencies $\Omega_{k_{j}}^{*}(j=1, \ldots, M)$, the landscape of the cost function (54) will be characterized, in general, by many local minima. Moreover, inspection of Eqs. (53) and (39) allows one to observe that the sensitivity of the cost function (54) with respect 
to the parameter $p$ tends to be substantially negligible for small values of the non-dimensional bending stiffness $\varepsilon$ typical of stay cables.

Gradient-based optimization algorithms, hence, are not well suited for the particular problem at hand, since they are prone to get trapped in local minima and their iteration operators could potentially be ill-conditioned for small values of $\varepsilon$. Within the class of gradient-free algorithms, the family of Differential Evolution (DE) algorithms, firstly proposed by Storn and Price [53], has shown excellent performances in finding the global optimum of non-linear, non-convex, multimodal and non-differentiable functions (see e.g. [14, 16]).

DE is an Evolutionary Algorithm that iteratively operates on a population of candidate solutions made of $N P$ parameter vectors. The initial population is randomly chosen within an initial searching volume $V_{0} \subseteq \mathcal{S}$ and offsprings are generated by perturbing trial solutions with scaled differences of randomly selected population elements. As the number of iterations grows, the characteristic size of these differences tend to automatically adapt to the natural scales of the objective landscape [16]. It is worth noting that the peculiar strategy adopted to generate offsprings and evolve the population of candidate solution makes DE algorithms able to deal with objective functions characterized by low or moderately low sensitivity with respect to one ore more variables of the searching space without numerical problems. Selection of the better fitted elements of the population is performed through a one-to-one parent/offspring competition scheme. The physical constraints can be enforced through a simple penalty criterion and the iterations are performed until a termination criterion is satisfied.

In the present work, a custom implementation of a well-known variant of the DE algorithm proposed by Das et al. [15] has been adopted to solve the non-linear optimization problem in Eq. (55). The termination criterion has been defined such that iterations are stopped whenever one of the following conditions is satisfied: (a) the relative difference between the best and worst objective function values $\Delta=\left(F_{\mathrm{obj}}^{\mathrm{worst}}-F_{\mathrm{obj}}^{\mathrm{best}}\right) / \max \left\{F_{\mathrm{obj}}^{\mathrm{worst}}, 1\right\}$ of a population is below a given threshold Toll (cf. the 'Diff' termination criterion proposed by Zielinski and Laur [61]), (b) the value of the cost function is lower than a prescribed value $F_{\mathrm{obj}}^{\mathrm{min}}$, (c) the number of iterations NIT is equal to a prescribed maximum number of iterations $M A X I T$.

Whenever multiple sets of observed frequencies are available, the non-linear optimization problem (55) can be repeatedly solved by means of independent runs of the DE algorithm. The average values and variances of the structural parameter estimates $\hat{\boldsymbol{X}}=\left(\hat{\Omega}_{0}, \hat{\varepsilon}, \hat{p}\right)^{T}$ and the combined uncertainty of the estimated axial force $\hat{T}$, then, can be calculated a posteriori, through a standard statistical analysis of the results of the identification procedure.

In order to better illustrate a typical run of the DE algorithm, a reference stay cable anchored to flexible supports and characterized by $\Omega_{0}=5.66 \mathrm{rad} / \mathrm{s}, \varepsilon=0.02$ and $p=0.25$ ("target values") is considered in the present section. The cost function (54) was defined by assuming that the first five natural frequencies of the system are known. Measured frequencies were numerically simulated by corrupting the theoretical results, obtained from the solution of the algebraic eigenvalue problem (21), through the addition of a small error term drawn from a zero-mean Gaussian distribution with coefficient of variation $I_{n}=0.5 \%$ (notice that $I_{n}$ can be conveniently regarded, within this context, as the measurement noise intensity). The optimization problem (55) has been solved by running the DE algorithm ("DEGL/SAW/bin" scheme, with scale factor $F=0.8$ and crossover parameter $C R=0.9$, see [15] for further details), starting from a population of $N P=30$ trial solutions randomly chosen in the initial search volume $V_{0}: 10^{-5} \leq \Omega_{0} \leq 50,10^{-5} \leq \varepsilon \leq 1,0 \leq p \leq 1$. The parameters of the termination criterion have been set up as: $F_{\mathrm{obj}}^{\min }=10^{-6}$, Toll $=10^{-7}$ and $M A X I T=5000$.

Figures 7 and 8 show the initial and final populations of candidate solutions, along with the result of the optimization run, i.e. the best member of the final population, and the target solution. The two figures allow one to clearly appreciate how the initial population, uniformly distributed in the initial searching volume $V_{0}$, evolves to a final population clustered in a small region of the searching space close to the target solution. It is also worth noting that the dispersion of the members of the final population with respect to variables $\Omega_{0}$ and $\varepsilon$ is much lower than the one with respect to $p$. This result is a direct consequence of the already mentioned low sensitivity of 


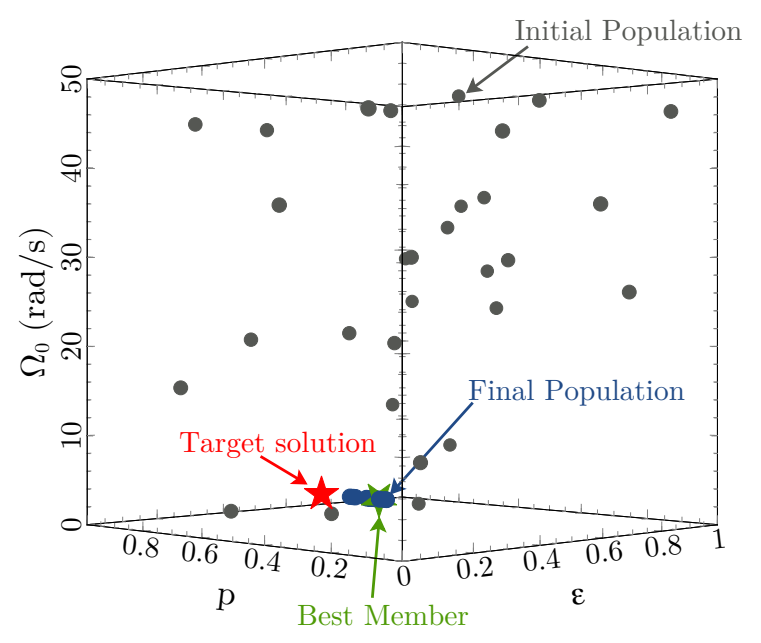

Figure 7: Illustration of a typical run of the DE algorithm. An initial population of $N P=30$ trial solutions randomly chosen in the initial search volume $V_{0}: 10^{-5} \leq \omega_{0} \leq 50,10^{-5} \leq \varepsilon \leq 1,0 \leq p \leq 1$ evolves to a final population close to the target solution characterized by the values: $\Omega_{0}=5.66 \mathrm{rad} / \mathrm{s}, \varepsilon=0.02$ and $p=0.25$

the cable natural frequencies with respect to the degree-of-fixity $p$.

Figure 9(a) shows the evolution of the best and worst values of the cost function (54) through the iterations of the DE algorithm. These two quantities converge to a common small value as the population of candidate solutions tends to concentrate in a neighborhood of the target solution. The components of the best parameter vector are shown in Figures 9(b), (c) and (d) as a function of the number of iterations. It is worth noting that parameters $\Omega_{0}$ and $\varepsilon$ rapidly converge to the corresponding target values. As expected, instead, variations of the parameter $p$ do not significantly affect the value of the cost function. This topic will be further discussed in Section 4.1

\subsection{Linear regression model}

Axial force identification strategies based on the numerical solution of a non-linear optimization problem, such as the one proposed in Section 3.2, can be computationally expensive and not well suited for structural health monitoring applications requiring continuous acquisition and on-line processing of experimental data. Furthermore, error propagation analyses can only be carried out through a posteriori statistical treatment of the outcomes of the identification procedure. This can lead to a considerable increase of the overall computational burden and prevents a deeper understanding of the effects on the axial force estimates of the main mechanical and geometrical parameters entering the structural model.

To circumvent these drawbacks, a novel approach is developed in the present Section for the simultaneous identification of the cable axial force and bending stiffness. The proposed procedure accounts for both the bending stiffness of the cable and the effects of the anchorage flexibility.It is based on the following steps: (a) a transformation of coordinates, mapping the non-linear asymptotic equation (53) into a linear one, (b) ordinary linear regression analysis. Once the regression coefficients are known, simple closed form equations allow one to get estimates of the characteristic frequency $\left(\hat{\Omega}_{0}\right)$ and non-dimensional bending stiffness $(\hat{\varepsilon})$ of the cable. Propagation of uncertainties is then investigated through approximate closed form equations, leading to a clear picture of the main geometrical and mechanical parameters affecting the results of the proposed identification procedure. 

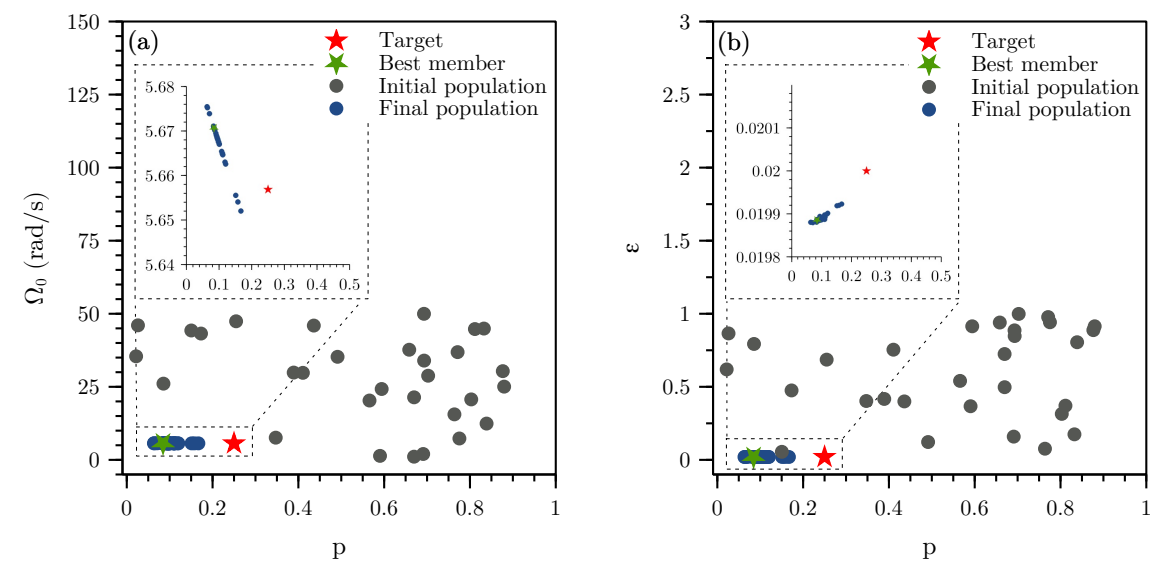

Figure 8: Illustration of a typical run of the DE algorithm. Projections of Figure 7 on the coordinate planes $\left(p, \Omega_{0}\right)$ (Figure $(\mathrm{a})$ ), and $(p, \varepsilon)$ (Figure $(\mathrm{b})$ ).
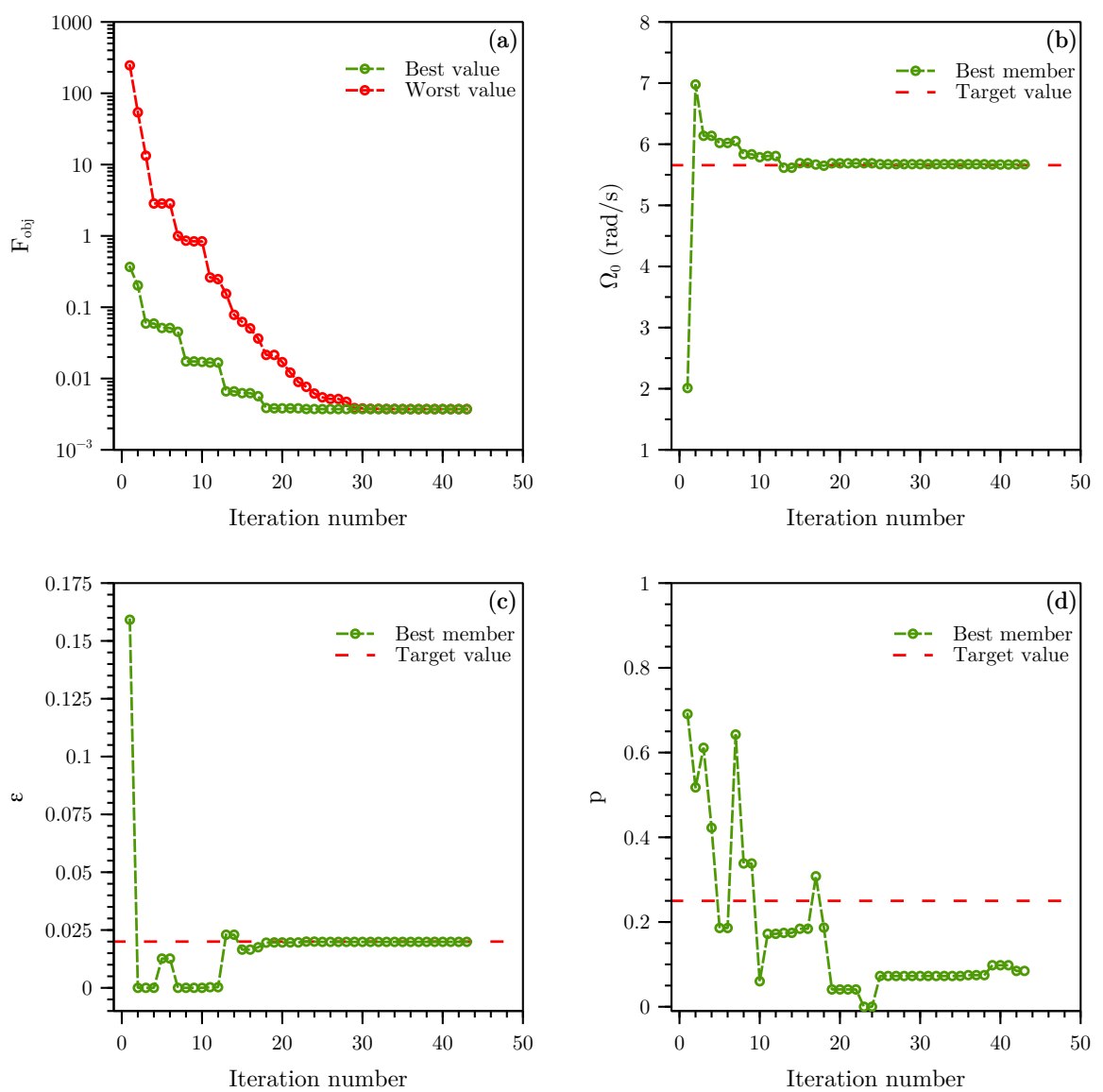

Figure 9: Illustration of a typical run of the DE algorithm. Figure (a) shows the best and worst values of the cost function (54) as a function of the number of iterations. Figures (b), (c) and (d) show the value of the components $\left(\Omega_{0}, \varepsilon, p\right)$ of the best parameter vector as a function of the number of iterations. 
Let us consider an ordered set of $M$ natural frequencies $\left\{\Omega_{k_{1}}, \Omega_{k_{2}}, \ldots, \Omega_{k_{M}}\right\}$, with $k_{1} \leq k_{2} \leq$ $\ldots \leq k_{M}\left(k_{j} \in \mathbb{N}^{+}, \forall j \in[1, M]\right)$, and introduce the integral coordinate

$$
\eta_{m}=\frac{1}{m} \sum_{j=1}^{m} \frac{\Omega_{k_{j}}}{\pi k_{j}}, \quad m=1, \ldots, M
$$

Substitution of (53) into this definition yields

$$
\eta_{m}=\frac{1}{m} \sum_{j=1}^{m} \frac{\pi k_{j} \Omega_{0}\left(1+f_{k_{j}}(\varepsilon, p)\right)}{\pi k_{j}}=\frac{\Omega_{0}}{m} \sum_{j=1}^{m}\left(1+2 p \varepsilon+\left(\frac{\left(k_{j} \pi\right)^{2}}{2}+4 p^{2}\right) \varepsilon^{2}\right),
$$

which can also be written

$$
\eta_{m}=\beta_{0}+\beta_{1} \gamma_{m}, \quad m=1, \ldots, M
$$

where

$$
\gamma_{m}=\frac{1}{m} \sum_{j=1}^{m} k_{j}^{2}
$$

and

$$
\beta_{0}=\Omega_{0}\left(1+2 p \varepsilon+4 p^{2} \varepsilon^{2}\right) \quad \text { and } \quad \beta_{1}=\Omega_{0} \frac{\varepsilon^{2} \pi^{2}}{2} .
$$

The use of this new coordinate shows that the original problem involving a quadratic sequence of the mode orders $\left\{k_{j}\right\}$ can be replaced by the simple linear relationship (59) in the new coordinate system $\left(\gamma_{m}, \eta_{m}\right)$.

Whenever a set of frequencies $\mathcal{M}^{*}=\left\{\Omega_{k_{1}}^{*}, \Omega_{k_{2}}^{*}, \ldots, \Omega_{k_{M}}^{*}\right\}$, associated to the modes $k_{1} \leq k_{2} \leq$ $\ldots \leq k_{M}$, is known from vibration tests, Eqs. (57) and (60) can be used to calculate the corresponding "experimental" points $\left(\gamma_{m}^{*}, \eta_{m}^{*}\right), m=1, \ldots, M$. Notice that the set $\left\{\eta_{1}, \ldots, \eta_{M}\right\}$ can also be regarded as sample of non-Gaussian random variable obtained by applying the non-linear transformation (57) to the set of independent Gaussian variables $\mathcal{M}=\left\{W_{k_{1}}, W_{k_{2}}, \ldots, W_{k_{M}}\right\}$, already introduced in Section 3.1. A full characterization of the probability density function of this resultant non-Gaussian random variable, although relatively straightforward, is outside the scope of the present work.

The coefficients $\beta_{0}$ and $\beta_{1}$, then, can be estimated through an application of the ordinary least squares method (see e.g. [57]):

$$
\begin{gathered}
\hat{\beta}_{1}=\frac{S_{\eta \gamma}}{S_{\gamma \gamma}} \\
\hat{\beta}_{0}=\bar{\eta}-\hat{\beta}_{1} \bar{\gamma}=\bar{\eta}-\frac{S_{\eta \gamma}}{S_{\gamma \gamma}} \bar{\gamma}
\end{gathered}
$$

where

$$
\bar{\eta}=\frac{1}{M} \sum_{m=1}^{M} \eta_{m}^{*}, \quad \bar{\gamma}=\frac{1}{M} \sum_{m=1}^{M} \gamma_{m}^{*}
$$

$$
S_{\eta \eta}=\frac{1}{M} \sum_{m=1}^{M}\left(\eta_{m}^{*}-\bar{\eta}\right)^{2}, S_{\gamma \gamma}=\frac{1}{M} \sum_{m=1}^{M}\left(\gamma_{m}^{*}-\bar{\gamma}\right)^{2}, S_{\eta \gamma}=\frac{1}{M} \sum_{m=1}^{M}\left(\eta_{m}^{*}-\bar{\eta}\right)\left(\gamma_{m}^{*}-\bar{\gamma}\right) .
$$

The estimators $\hat{\beta}_{0}$ and $\hat{\beta}_{1}$ given by these formulae are also available in any commercial software as a standard tool of basic fitting. The proposed linear fitting has therefore no specific difficulty, other than the establishment of the transformed coordinates $(\eta, \gamma)$. Figure 10 illustrates a typical application of the coordinate transformation defined by Eqs. (57) and (60) to the first five natural frequencies of a cable characterized by $\Omega_{0}=5.66 \mathrm{rad} / \mathrm{s}, \varepsilon=0.02$ and $p=0.5$. Ten samples of measured frequencies have been numerically simulated by corrupting the theoretical results of the 

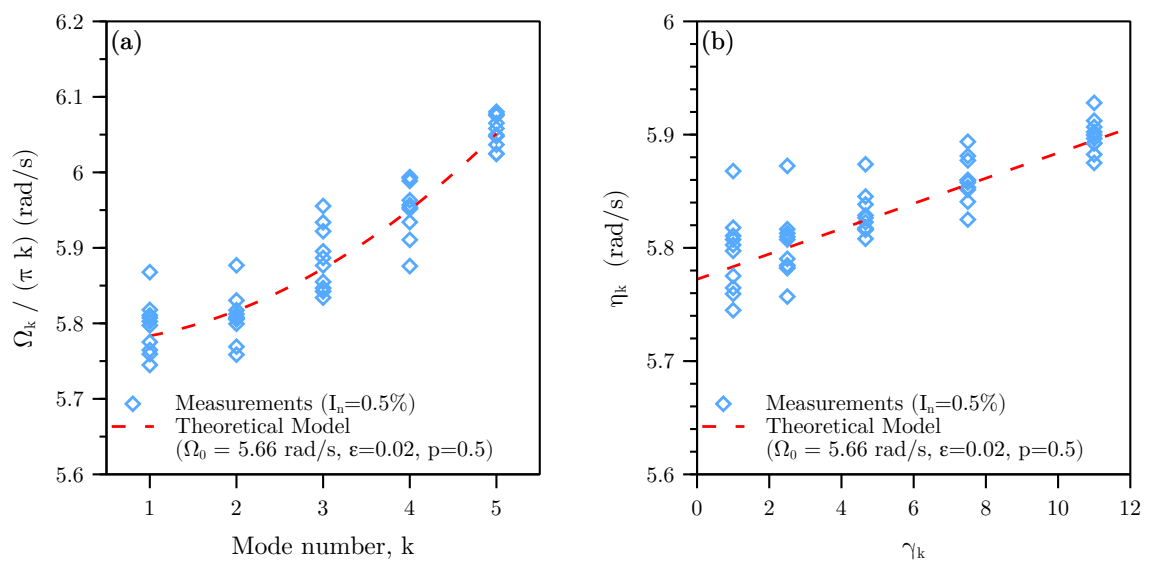

Figure 10: First five natural frequencies of a stay cable characterized by $\Omega_{0}=5.66 \mathrm{rad} / \mathrm{s}, \varepsilon=0.02$ and $p=0.5$. Measured results are corrupted by a zero-mean Gaussian noise with intensity $I_{n}=0.5 \%$. (a) Representation in the plane $\left(k, \frac{\Omega_{k}}{k \pi}\right)$. (b) Representation in the plane $\left(\gamma_{k}, \eta_{k}\right)$, obtained through the coordinate transformation defined in Eqs. (57) and (60).

semi-analytical model, presented in Section 2.3, through the addition of a low-intensity zero-mean Gaussian noise. Figures 10(a) and 10(b) respectively show the simulated measurement results and their transformed representation in the coordinate system $\left(\gamma_{m}, \eta_{m}\right)$. The outcomes of the underlying theoretical (i.e. free from noise) model are also shown for comparison purposes (red dashed lines).

Once $\hat{\beta}_{0}$ and $\hat{\beta}_{1}$ are determined, substitution of $\hat{\beta}_{0}$ and $\hat{\beta}_{1}$ in Eqs. (61) yields, after some rearrangements, the system of equations

$$
\left\{\begin{array}{l}
\frac{\hat{\varepsilon}^{2}}{1+2 p \hat{\varepsilon}+4 p^{2} \hat{\varepsilon}^{2}}=\frac{2}{\pi^{2}} \hat{\beta}^{2} \\
\hat{\beta}_{0} \\
\hat{\Omega}_{0}\left(1+2 p \hat{\varepsilon}+4 p^{2} \hat{\varepsilon}^{2}\right)=\hat{\beta}_{0}
\end{array}\right.
$$

where $\hat{\Omega}_{0}$ and $\hat{\varepsilon}$ are estimates of the characteristic frequency and the non-dimensional stiffness of the cable.

Equations (61) and (66) allow one to notice that the intercept $\beta_{0}$ of the linear regression model is of the same order of magnitude as $\Omega_{0}$, while the slope $\beta_{1}$ is of the same order as $\varepsilon^{2}$. For typical stay cables, $\varepsilon \ll 1$ and $\Omega_{0} \sim 1$. A first-order accurate approximate solution of (66), hence, can be expressed as

$$
\begin{gathered}
\hat{\Omega}_{0}=\hat{\beta}_{0}-p \frac{2 \sqrt{2}}{\pi} \sqrt{\hat{\beta}_{0} \hat{\beta}_{1}} \\
\hat{\varepsilon}=\frac{\sqrt{2}}{\pi} \sqrt{\frac{\hat{\beta}_{1}}{\hat{\beta}_{0}}}
\end{gathered}
$$

Once $\hat{\Omega}_{0}$ and $\hat{\varepsilon}$ are known, estimates of the cable axial force $(\hat{T})$ and bending stiffness $(\hat{E} I)$ can be respectively obtained through Eqs. (44) and (56).

Substitution of Eq. (68) in (67) yields the following, more expressive, equation for $\hat{\Omega}_{0}$

$$
\hat{\Omega}_{0}=\hat{\beta}_{0}(1-2 p \hat{\varepsilon})
$$

Inspection of Eqs. (68) and (69) allows one to observe that modeling errors on the boundary conditions do not affect $\hat{\varepsilon}$, but can introduce a bias on the estimator $\hat{\Omega}_{0}$.

It is interesting to notice that, at first order, the linear relation between $\eta_{m}$ and $\gamma_{m}$ expressed in (59) just depends on the two parameters $\beta_{0}$ and $\beta_{1}$. This indicates that it is impossible to 
independently determine the three parameters $\Omega_{0}, \varepsilon$ and $p$. In principle, a third equation for the third unknown could be determined by considering the governing equations at second order. This investigation track (to form a well-posed set of equations) is not further developed because the order of magnitude of the second-order terms are very likely to fall within the measurement noise in most practical cases. Instead, in the solutions expressed in (67-68) we have considered $p$ as a parameter.

Let us denote as $p^{*}$ an assumed value of the restraint parameter $p$ that, after substitution in Eq. (69), allows to get $\hat{\Omega}_{0}$ for given $\hat{\varepsilon}$ and $\hat{\beta}_{0}$. Moreover, let us define as $\Delta p=p^{*}-p$ the difference between the assumed $\left(p^{*}\right)$ and the unknown "true" value $(p)$ of the restraint parameter. A first order accurate estimate expression of the bias of the estimator $\hat{\Omega}_{0}$ can be easily obtained from Eq. (69)

$$
\operatorname{bias}\left[\hat{\Omega}_{0}\right]=2 \Delta p \hat{\varepsilon} \hat{\Omega}_{0} .
$$

By assuming $p^{*}=0$, i.e. by modeling both cable restraints as perfect hinges, one gets $\Delta p=-p$ and Eq. (70) approximately coincides with the first order term in the Eq. (47), describing the bias of the identification procedure based on the taut string model. Furthermore, whenever the translational flexibility of the cable anchorages is negligible, the "true" value of the degree-of-fixity parameter can only vary in the range $0 \leq p \leq 1$. As a consequence, the bias of the estimator $\hat{\Omega}_{0}$ turns out to be bounded, with upper and lower bound values implicitly defined by Eq. (70). For example, by pragmatically assuming the intermediate value $p^{*}=0.5$, Eq. (70) yields

$$
\left|\frac{1}{\hat{\Omega}_{0}} \operatorname{bias}\left[\hat{\Omega}_{0}\right]\right| \leq \hat{\varepsilon}
$$

By denoting respectively as $\sigma_{\hat{\beta}_{0}}^{2}, \sigma_{\hat{\beta}_{1}}^{2}$ and $\rho_{\hat{\beta}_{0} \hat{\beta}_{1}}$ the variances and correlation coefficient of the regression parameters $\hat{\beta}_{0}$ and $\hat{\beta}_{1}$, a standard linearized error propagation model (see e.g. [5]) allows to approximately evaluate the variances of $\hat{\Omega}_{0}$ and $\hat{\varepsilon}$ as

$$
\sigma_{\hat{\Omega}_{0}}^{2}=\left(\frac{\partial \hat{\Omega}_{0}}{\partial \hat{\beta}_{0}}\right)^{2} \sigma_{\hat{\beta}_{0}}^{2}+\left(\frac{\partial \hat{\Omega}_{0}}{\partial \hat{\beta}_{1}}\right)^{2} \sigma_{\hat{\beta}_{1}}^{2}+\frac{\partial \hat{\Omega}_{0}}{\partial \hat{\beta}_{0}} \frac{\partial \hat{\Omega}_{0}}{\partial \hat{\beta}_{1}} \rho_{\hat{\beta}_{0} \hat{\beta}_{1}} \sigma_{\hat{\beta}_{0}} \sigma_{\hat{\beta}_{1}}
$$

and

$$
\sigma_{\hat{\varepsilon}}^{2}=\left(\frac{\partial \hat{\varepsilon}}{\partial \hat{\beta}_{0}}\right)^{2} \sigma_{\hat{\beta}_{0}}^{2}+\left(\frac{\partial \hat{\varepsilon}}{\partial \hat{\beta}_{1}}\right)^{2} \sigma_{\hat{\beta}_{1}}^{2}+\frac{\partial \hat{\varepsilon}}{\partial \hat{\beta}_{0}} \frac{\partial \hat{\varepsilon}}{\partial \hat{\beta}_{1}} \rho_{\hat{\beta}_{0} \hat{\beta}_{1}} \sigma_{\hat{\beta}_{0}} \sigma_{\hat{\beta}_{1}}
$$

Equations (72) and (73) rely on the assumption of small coefficients of variation $\frac{\sigma_{\hat{\beta}_{0}}}{\hat{\beta}_{0}}$ and $\frac{\sigma_{\hat{\beta}_{1}}}{\hat{\beta}_{1}}$, i.e. $\frac{\sigma_{\hat{\beta}_{0}}}{\hat{\beta}_{0}} \ll 1, \frac{\sigma_{\hat{\beta}_{1}}}{\hat{\beta}_{1}} \ll 1$. As it will be further shown in Section 4.2 through numerical examples, typical values of the the ratio $\frac{\sigma_{\hat{\beta}_{0}}}{\hat{\beta}_{0}}$ are in the order of a few percent, while the order of magnitude of the coefficient of variation $\frac{\sigma_{\hat{\beta}_{1}}}{\hat{\beta}_{1}}$ can rapidly approach the unity for decreasing values of the non-dimensional bending stiffness $\varepsilon$ and increasing values of the noise intensity.

Although approximate, Eqs. (72) and (73) allows one to gain a deeper insight on the propagation of uncertainties within the proposed identification procedure. By assuming that $\frac{\sigma_{\hat{\beta}_{0}}}{\hat{\beta}_{0}} \sim \hat{\varepsilon} \frac{\sigma_{\hat{\beta}_{1}}}{\hat{\beta}_{1}}$, substitution of the partial derivatives of $\hat{\Omega}_{0}$ and $\hat{\varepsilon}$ in Eqs. (72) and (73) yields, after some simple rearrangements, the following second-order accurate approximate equations

$$
\begin{aligned}
& \left(\frac{\sigma_{\hat{\Omega}_{0}}}{\hat{\Omega}_{0}}\right)^{2}=\left(\frac{\sigma_{\hat{\beta}_{0}}}{\hat{\beta}_{0}}\right)^{2}-p \hat{\varepsilon} \rho_{\hat{\beta}_{0} \hat{\beta}_{1}} \frac{\sigma_{\hat{\beta}_{0}}}{\hat{\beta}_{0}} \frac{\sigma_{\hat{\beta}_{1}}}{\hat{\beta}_{1}} p \hat{\varepsilon}^{2}\left(\frac{\sigma_{\hat{\beta}_{1}}}{\hat{\beta}_{1}}\right)^{2} \\
& \left(\frac{\sigma_{\hat{\varepsilon}}}{\hat{\varepsilon}}\right)^{2}=\frac{1}{4}\left[\left(\frac{\sigma_{\hat{\beta}_{1}}}{\hat{\beta}_{1}}\right)^{2}-\rho_{\hat{\beta}_{0} \hat{\beta}_{1}} \frac{\sigma_{\hat{\beta}_{0}}}{\hat{\beta}_{0}} \frac{\sigma_{\hat{\beta}_{1}}}{\hat{\beta}_{1}}+\left(\frac{\sigma_{\hat{\beta}_{0}}}{\hat{\beta}_{0}}\right)^{2}\right] .
\end{aligned}
$$


Equation (74) clearly allows one to appreciate that the leading order term of the coefficient of variation of $\hat{\Omega}_{0}$ is equal to that of $\hat{\beta}_{0}$. Correction terms increase with $\hat{\varepsilon}$ and are linearly proportional to the value of the restraint parameter $p$. On the other hand, Eq. (75) shows that the coefficient of variation of $\hat{\varepsilon}$ is independent of the boundary conditions and with a leading order term equal to $\frac{1}{2} \frac{\sigma_{\hat{\beta}_{1}}}{\hat{\beta}_{1}}$.

In summary, in this Section, we have proposed an extension of the identification procedure for a cable with a focus on the influence of unknown end conditions. By exploiting the asymptotic response for small bending stiffness, we have shown that it is only possible to determine two of the three parameters $\Omega_{0}, \varepsilon$ and $p$ with the help of a simple linear regression model. For practical reasons, parameter $p$ is typically difficult to determine, a reason why we have suggested to consider it as a known parameter of the model, since, in most cases of practical interest $p \in[0,1]$, a pragmatical choice $p=0.5$ could be formulated. It lessens the bias and standard error on the estimate that could be obtained by assuming hinged-hinged end conditions. More importantly, the bias on the estimated cable tension could be bounded by considering the two limit cases $p=0$ and $p=1$. Finally, because of the simplicity of this asymptotic model, the uncertainty propagation analysis of the proposed identification procedure could be derived. The main trends indicating in which way the problem parameters do affect the standard errors of the estimated cable tension and bending stiffness could be determined. These will be illustrated in the following Section.

\section{Applications}

The performances of the novel identification strategies described in Sections 3.2 and 3.3 have been assessed through extensive numerical testing. The results will be presented in the following with reference to a stay cable attached to anchorages with negligible translational flexibility and characterized by $\Omega_{0}=5.66 \mathrm{rad} / \mathrm{s}$ and $T=4000 \mathrm{kN}$. Three different values of non-dimensional bending stiffness $(\varepsilon=0.01,0.02,0.03)$ have been considered, along with five different boundary conditions: $\left(\mathrm{I}_{R}\right) p=0$ (doubly-hinged cable), $\left(\mathrm{II}_{R}\right) p=0.25,\left(\mathrm{III}_{R}\right) p=0.50,\left(\mathrm{IV}_{R}\right) p=0.75$ and $\left(\mathrm{V}_{R}\right) p=1$ (doubly-clamped cable).

In order to simulate experimental input data, the algebraic eigenvalue problem (21) has been numerically solved to get the first five natural frequencies of the system. These reference values, then, have been corrupted through multiplication by a unit-mean and low intensity Gaussian noise, to account for the effects of measurement errors. Different values of noise intensity, ranging from 0 to $2.5 \%$, have been considered. Please notice that the range of noise intensity values herein considered is consistent with the expected outcome of standard dynamic testing techniques for the identification of natural frequencies of stay cables (see e.g. [49, 54]). For each noise intensity value, a sample of 1000 sets of noisy natural frequencies has been independently randomly generated.

Sections 4.1 and 4.2 present the results of the proposed identification strategies based on, respectively, the non-linear optimization problem (see Section 3.2) and the linear regression model (see Section 3.3). Section 4.3 reports comparisons between the outcomes of the proposed identification strategies and the ones of the classic approach relying on the taut string model (see Section $3.1)$.

\subsection{Solution of the non-linear optimization problem}

The non-linear optimization problem (55) has been solved by running the DE algorithm presented in Section 3.2 ("DEGL/SAW/bin" scheme, with scale factor $F=0.8$ and crossover parameter $C R=0.9$, see [15] for further details), starting from a population of $N P=30$ trial solutions randomly chosen in the initial search volume $V_{0}: 10^{-5} \leq \Omega_{0} \leq 50,10^{-5} \leq \varepsilon \leq 1,0 \leq p \leq 1$. The parameters of the termination criterion have been set up as: $F_{o b j}^{\min }=10^{-6}$, Toll $=10^{-7}$ and $M A X I T=5000$. Please notice that the initial search space $V_{0}$ satisfies all physical constraints of the structural problem (i.e. $\Omega_{0}>0, \varepsilon>0$ and $0 \leq p \leq 1$ ). Since trial solutions are not constrained within the initial search volume $V_{0}$, however, no special criteria needs to be adopted to define $V_{0}$. As a consequence, the initial upper bounds values of the variables $\Omega_{0}$ and $\varepsilon$ have been herein defined in order to be large enough to highlight the good convergence properties of 
the optimization algorithm also whenever initial guesses for these structural parameters are not available.

Figure 11 shows the results of the identification procedure, as a function of the noise intensity, for a stay cable with target bending stiffness value $\varepsilon=0.02$. Results are averaged over the number of runs $N R$ of the algorithm (i.e. $N R=1000$ ) for every different noise intensity level and expressed in terms of: (a-c) the identified values of the parameters $\hat{\Omega}_{0, a v e}, \hat{\varepsilon}_{\text {ave }}$ and $\hat{p}_{\text {ave }}$; (d) the relative error on the element axial force, $\Delta T=\frac{\hat{T}_{\text {ave }}-T_{\text {target }}}{T_{\text {target }}}$, where $\hat{T}_{\text {ave }}$ is the average identified value of the axial force and $T_{\text {target }}$ is the target value (i.e. $T_{\text {target }}=4000 \mathrm{kN}$ ); (e) $F_{\text {obj,ave }}^{\text {best }}$ is the average value of the cost function associated to the identified optimal set of parameters; (f) $N I T_{\text {ave }}$ is the average number of iterations of the DE algorithm.

The identification strategy gives fairly accurate results in terms of parameters $\Omega_{0}$ and $\varepsilon$ for all values of noise intensity herein considered. As already noticed in Section 3.1, the boundary conditions do not significantly affect the cost function (54) and, as a consequence, the identification algorithm is not able to correctly identify the parameter $p$. For each value of noise intensity, the identification procedure tends to a mean value of $p$ equal to about 0.5 , no matter the target value in $[0,1]$. This mean value actually coincides with the mean value of $p$ within the randomly generated candidate solutions of the DE algorithm.

On the overall, in spite of a poor identification of the degree-of-fixity $p$ of the beam end sections, the procedure gives a good estimate of the axial force. Errors on the average identified axial force value are not substantially affected by noise intensity and, since $p \rightarrow 0.5$, the bias is higher for the two boundary conditions corresponding to the limit cases of doubly-hinged (label $\mathrm{I}_{R}, p=0$ ) and doubly-clamped (label $\mathrm{V}_{R}, p=1$ ) cables, for which $|\Delta p|=0.5$, see (70).

Figure 11(e) allows to appreciate how measurements errors, herein associated to non-zero noise intensity values, determine a significant jump in the estimated minimum values of the cost function. Figure 11(f) shows that convergence of the algorithm is usually reached after a number of iterations significantly lower than the prescribed maximum number MAXIT, because the relative difference between the best and worst objective function is lower than the prescribed tolerance value (see the description of the termination criterion in Section 3.1).

As it can be clearly appreciated from Figure 11(a), errors on the parameter $p$ introduce a bias on the estimates of $\hat{\Omega}_{0}$. Numerical tests carried out for different values of $\varepsilon$, in the typical range of values of stay cables, have shown that the bias of the estimator $\hat{\Omega}_{0}$ is very well approximated by the same Eq. (70) originally derived for the linear regression model. The analysis of the outcomes of the DE algorithm, hence, suggests that, whenever in presence of cable anchorages with unknown rotational flexibility, a pragmatical approach to reduce the computational burden of the identification procedure without affecting the average values of the estimated parameters $\hat{\Omega}_{0}$ and $\hat{\varepsilon}_{0}$ is to assume $p=0.5$. Under this assumption, the maximum expected relative error on $\hat{\Omega}_{0}$ turns out to be in the range $\pm \hat{\varepsilon}$ (cf. Eq. (71)).

Figure 12 shows a comparison between the results of the standard DE algorithm presented in Section 3.2 and the one based on the pragmatical assumption $p=0.5$, reducing therefore the searching space to a two-dimensional space. In all cases, computations have been performed by using the control parameters of the DE algorithm previously reported in this Section and the outcomes averaged over the number of runs $N R=1000$ for each noise intensity level. Reference stay cables with the same boundary conditions $(p=0.5)$ and three different values of $\varepsilon(\varepsilon=0.01$, 0.02 and 0.03 ) have been considered. The results are shown in terms of the average values of $\hat{\Omega}_{0}$ and $\hat{\varepsilon}$ (Figures 12(a) and 12(c)) along with their associated coefficient of variation (Figures 12(b) and 12(d)). As it can be appreciated from Figure 12, the outcomes of the two-parameter identification algorithm based on the pragmatical assumption $p=0.5$ are practically coincident with the ones of the three-parameter DE algorithm, with an important difference for the coefficient of variation of $\hat{\Omega}_{0}$. Enforcing the constraint $p=0.5$, indeed, leads to values of $\sigma_{\hat{\Omega}_{0}} / \hat{\Omega}_{0}$ that are independent of $\varepsilon$ and well approximated by the linear relation $\sigma_{\hat{\Omega}_{0}} / \hat{\Omega}_{0}=I_{n}$. These values are in general smaller than the ones coming from the three-parameter DE algorithm and shows the same linear trend as the classic identification strategy based on the taut string model, see Eq. (51). 

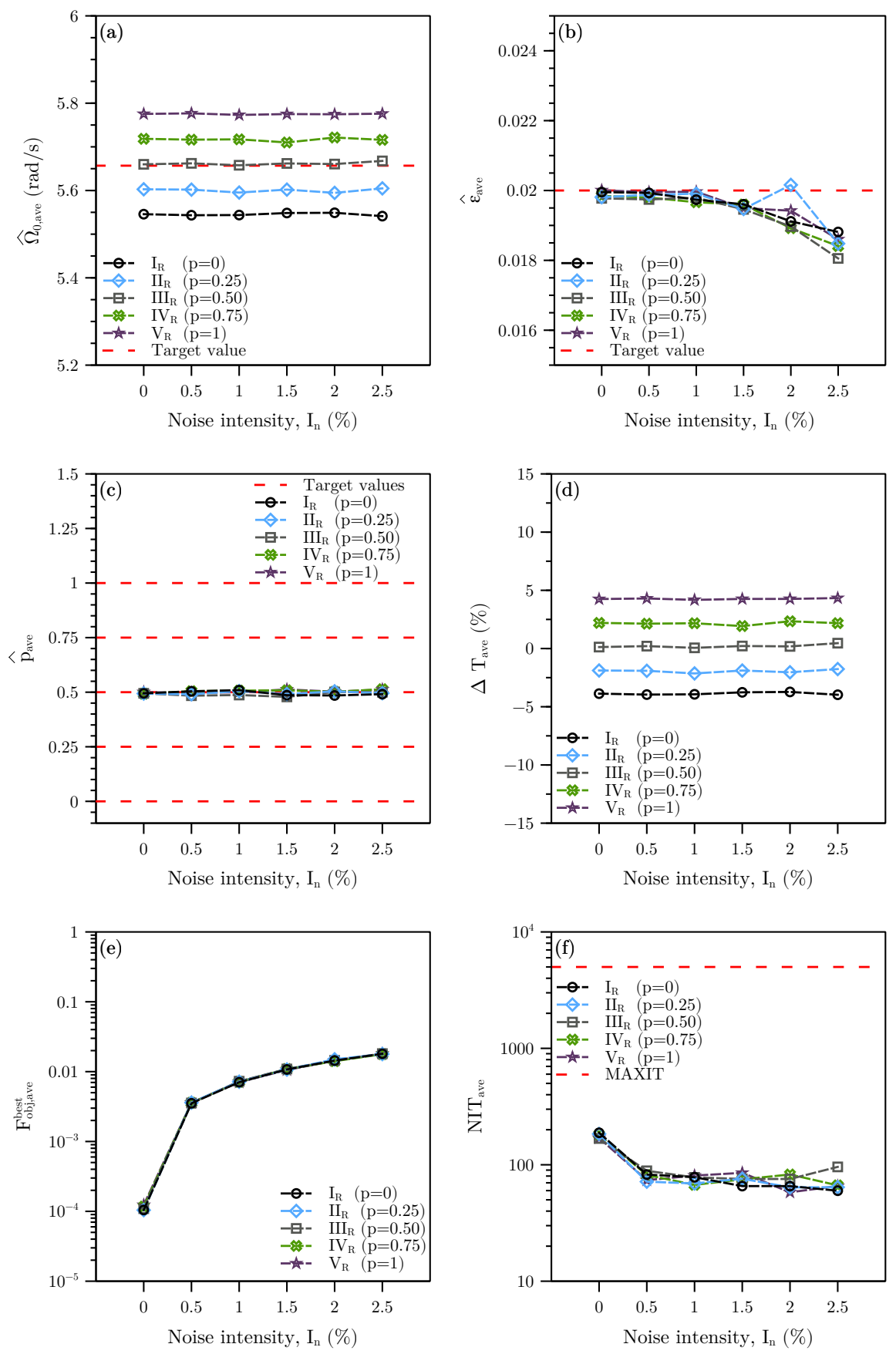

Figure 11: Non-linear optimization problem. Results of the Differential Evolution (DE) algorithm, averaged over one-thousand runs, as a function of the noise intensity. (a) Characteristic frequency $\Omega_{0}$ (target value: $\Omega_{0}=5.66$ $\mathrm{rad} / \mathrm{s}$ ). (b) Non-dimensional bending stiffness $\varepsilon$ (target value: $\varepsilon=0.02$ ). (c) Degree-of-fixity parameter $p$. (d) Relative error on the axial force $\Delta T=\frac{\hat{T}-T_{\text {target }}}{T_{\text {target }}}$. (e) Value of the cost function associated to the identified optimal set of parameters. (f) Number of iterations (the maximum number is $M A X I T=5000$ ). 

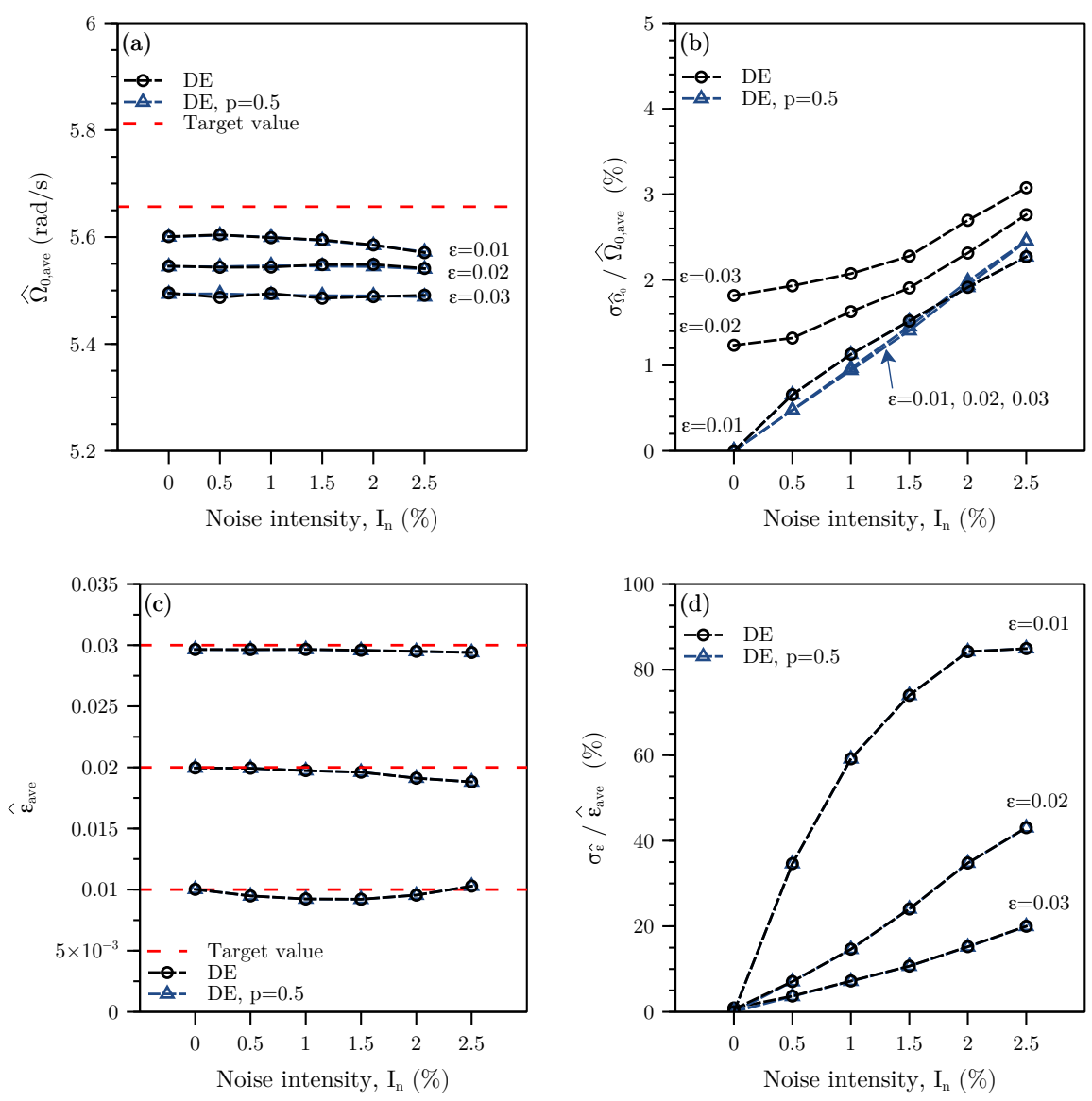

Figure 12: Non-linear optimization problem. Comparison among the results of the Differential Evolution algorithm with unknown parameters: $\Omega_{0}, \varepsilon$ and $p(\mathrm{DE})$ and the ones obtained under the assumption $p=0.5(\mathrm{DE}, p=0.5)$. The results are averaged over one-thousand runs and shown as a function of the noise intensity. (a) Characteristic frequency $\Omega_{0}$ (target value: $\Omega_{0}=5.66 \mathrm{rad} / \mathrm{s}$ ). (b) Coefficient of variation of the estimated value of $\Omega_{0}$. (c) Nondimensional bending stiffness $\varepsilon$ (target values: $\varepsilon=0.01,0.02$ and 0.03 ). (d) Coefficient of variation of the estimated value of $\varepsilon$. 


\subsection{Results of the linear regression model}

As a first illustration, the linear regression model presented in Section 3.3 is applied to a stay cable characterized by target bending stiffness $\varepsilon=0.02$ and restraint parameter $p=0.5$ (cf. the boundary condition labeled as $I I I_{R}$ in Section 4.1). The results of the identification algorithm are averaged over the number of runs $N R=1000$ for each noise intensity level. Figure 13 shows the average values of $\hat{\Omega}_{0}$ and $\hat{\varepsilon}$, (a) and (c), along with their associated coefficient of variations, (b) and (d). Figures 13(a) and 13(b) report, for comparison purposes, the results obtained by setting the restraint parameter $p$ in Eq. (69) equal to: $p=0$ (curves labeled as " $L R, p=0$ "), $p=0.5$ (" $L R, p=0.5 ")$ and $p=1$ (" $L R, p=1$ "). Different assumptions on $p$ do not affect the estimate $\hat{\varepsilon}$ of the non-dimensional bending stiffness (see Eqs. (68) and (75)). As a consequence, a single curve labeled as " $L R$ " reports the results of the proposed identification procedure in Figures 13(c) and 13(d). The outcomes of the approximate closed-form equations (74) and (75) for the coefficients of variation $\sigma_{\hat{\Omega}_{0}} / \hat{\Omega}_{0}$ and $\sigma_{\hat{\varepsilon}} / \hat{\varepsilon}$ are also reported in Figures 13(b) and 13(d) (see the dashed curves, labeled as "Approx. Model"). Figures 14(a) and 14(b) respectively show the coefficient of variation of the linear regression parameters $\hat{\beta}_{0}$ and $\hat{\beta}_{1}$ as a function of the noise intensity.

Figure 13(a) shows that the average values of $\hat{\Omega}_{0}$ are substantially independent of the noise intensity and biased by modeling errors on the boundary conditions, herein globally modeled though the restraint parameter $p$. As expected from Eq. (70), bias-induced discrepancies between the estimated and target values of the characteristic frequency $\Omega_{0}$ are practically negligible for the model " $L R, p=0.5$ ", while relative errors equal to about $+\varepsilon$ and $-\varepsilon$ (i.e. $\pm 2 \%$ ) are respectively associated to the models " $L R, p=0$ " and " $L R, p=1$ ".

As it can be appreciated from Figure 13(b), the coefficient of variation $\sigma_{\hat{\Omega}_{0}} / \hat{\Omega}_{0}$ is almost linearly related to the noise noise intensity $I_{n}$. Slope values increase with the restraint parameter, ranging from about 0.9 for $p=0$ to about 1.7 for $p=1$. The numerical results shown in Figure 13(b) are practically coincident with the outcomes of the approximate closed-form error propagation Eq. (74) for the special case $p=0$ (i.e. doubly-hinged stay cable). Discrepancies between the numerical results and the outcomes of the approximate model increase with the increase of both $p$ and the noise intensity. The reason for these discrepancies can be traced back to the behavior of the coefficient of variation of the linear regression coefficient $\hat{\beta}_{1}$, which satisfies the considered assumption $\sigma_{\hat{\beta}_{1}} / \hat{\beta}_{1} \ll 1$ only for small values of the noise intensity, as it can be clearly observed from Figure 14(b).

The proposed identification procedure also delivers fairly good estimates of the non-dimensional bending stiffness $\varepsilon$, as it can be appreciated from Figure 13(c). Discrepancies between the average value of $\hat{\varepsilon}$ and the target value $\varepsilon=0.02$ increase with the noise intensity, ranging from about $1.8 \%$ for $I_{n}=0$ to about $11 \%$ for $I_{n}=2.5 \%$. It is worth noting that the small error corresponding to the ideal case of experimental data free from noise (i.e. $I_{n}=0$ ) can be regarded as a modeling error due to the adoption of the asymptotic closed form Eq. (37) instead of the exact solution of the semi-analytical model presented in Section 2.3.

The values of the coefficient of variation $\sigma_{\hat{\varepsilon}} / \hat{\varepsilon}$ increase with the noise intensity, as it is shown in Figure 13(d). A comparison between Figures 13(d) and 14(b) clearly allows one to appreciate that $\sigma_{\hat{\varepsilon}} / \hat{\varepsilon}$ is mainly governed by the leading order term of the approximate equation (75), i.e.: $\frac{1}{2} \frac{\sigma_{\hat{\beta}_{1}}}{\hat{\beta}_{1}}$. It can also be observed how the closed form Eq. (75) delivers a reasonably good approximation of $\sigma_{\hat{\varepsilon}} / \hat{\varepsilon}$ for small values of noise intensity, with discrepancies in the order of about $15 \%$ and $30 \%$ for $I_{n}$ respectively equal to $1 \%$ and $2.5 \%$.

Parametric analyses have been carried out to assess the performance of the linear regression model for different values of the non-dimensional bending stiffness $\varepsilon$. Figure 15 depicts the outcomes of the proposed identification procedure for three stay cables characterized by $\Omega_{0}=5.66$ $\mathrm{rad} / \mathrm{s}, p=0.5$ and different values of $\varepsilon$, i.e. $\varepsilon=0.01,0.02$ and 0.03 . The linear regression model has been applied by setting the restraint parameter $p$ in Eq. (69) equal to $p=0.5$, i.e. by modeling boundary conditions without errors. The results of the identification algorithm are averaged over the number of runs $N R$ (i.e. $N R=1000$ ) for each noise intensity level.

The average values of $\hat{\Omega}_{0}$ (Figure 15(a)) are substantially independent of $\varepsilon$ and unbiased, according to the predictions of Eq. (70). Furthermore, the coefficient of variation $\sigma_{\hat{\Omega}_{0}} / \hat{\Omega}_{0}$ turns 

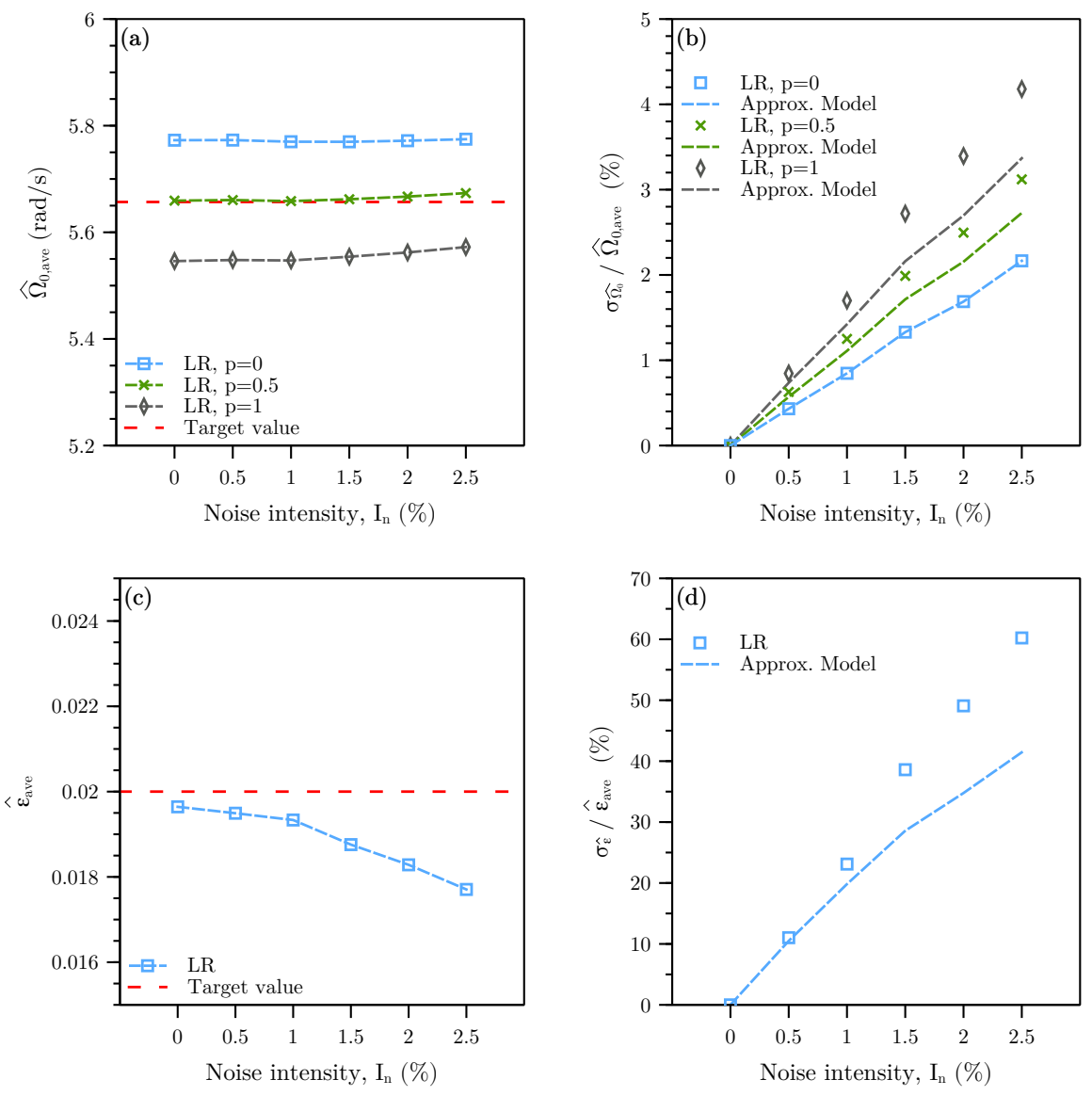

Figure 13: Results of the linear regression model, averaged over one-thousand runs, as a function of the noise intensity for a stay cable anchored to flexible restraints characterized by a theoretical value of the restraint parameter equal to $p=0.5$. (a) Characteristic frequency $\Omega_{0}$ (target value: $\Omega_{0}=5.66 \mathrm{rad} / \mathrm{s}$ ). (b) Coefficient of variation of the estimated value of $\Omega_{0}$. (c) Non-dimensional bending stiffness $\varepsilon$ (target value: 0.02 ). (d) Coefficient of variation of the estimated value of $\varepsilon$. The results shown in Figures 13(a) and 13(b) have been obtained by setting the restraint parameter $p$ in Eq. (69) equal to: $p=0$ (curves labeled as $L R, p=0), p=0.5(L R, p=0.5)$ and $p=1(L R, p=1)$. Approximate results (labeled as Approx. Model) in Figures (b) and (d) are respectively obtained through Eqs. (74) and (75). 

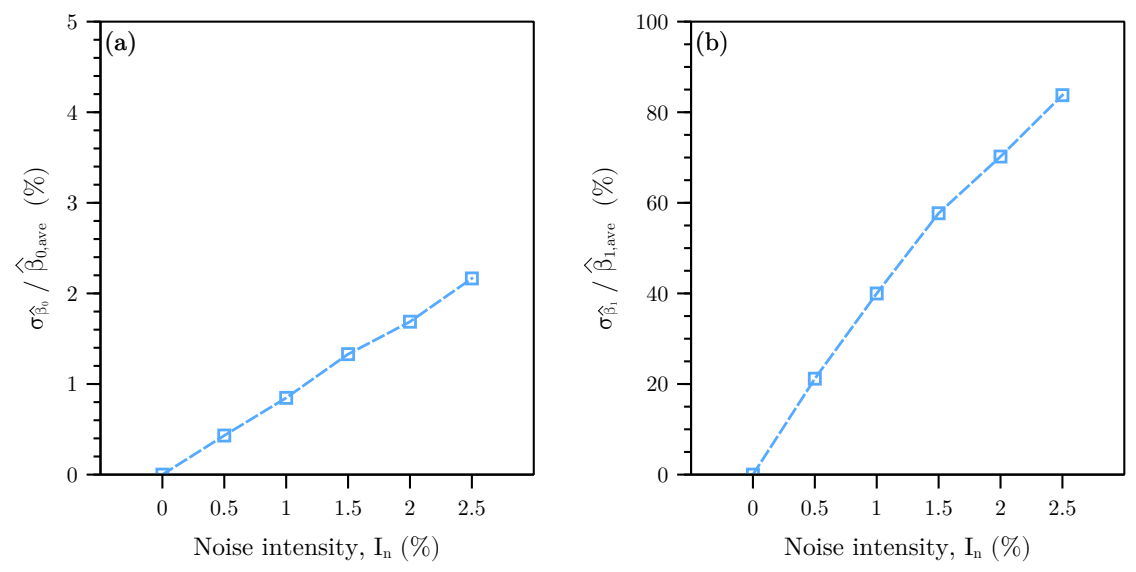

Figure 14: Results of the linear regression model, averaged over one-thousand runs, as a function of the noise intensity for a stay cable anchored to flexible restraints characterized by: $\Omega_{0}=5.66 \mathrm{rad} / \mathrm{s}, \varepsilon=0.02$ and $p=0.5$. Coefficients of variation of the linear regression parameters: (a) $\hat{\beta}_{0}$ (see Eq. (63)), and (b) $\hat{\beta}_{1}$ (see Eq. (62)).
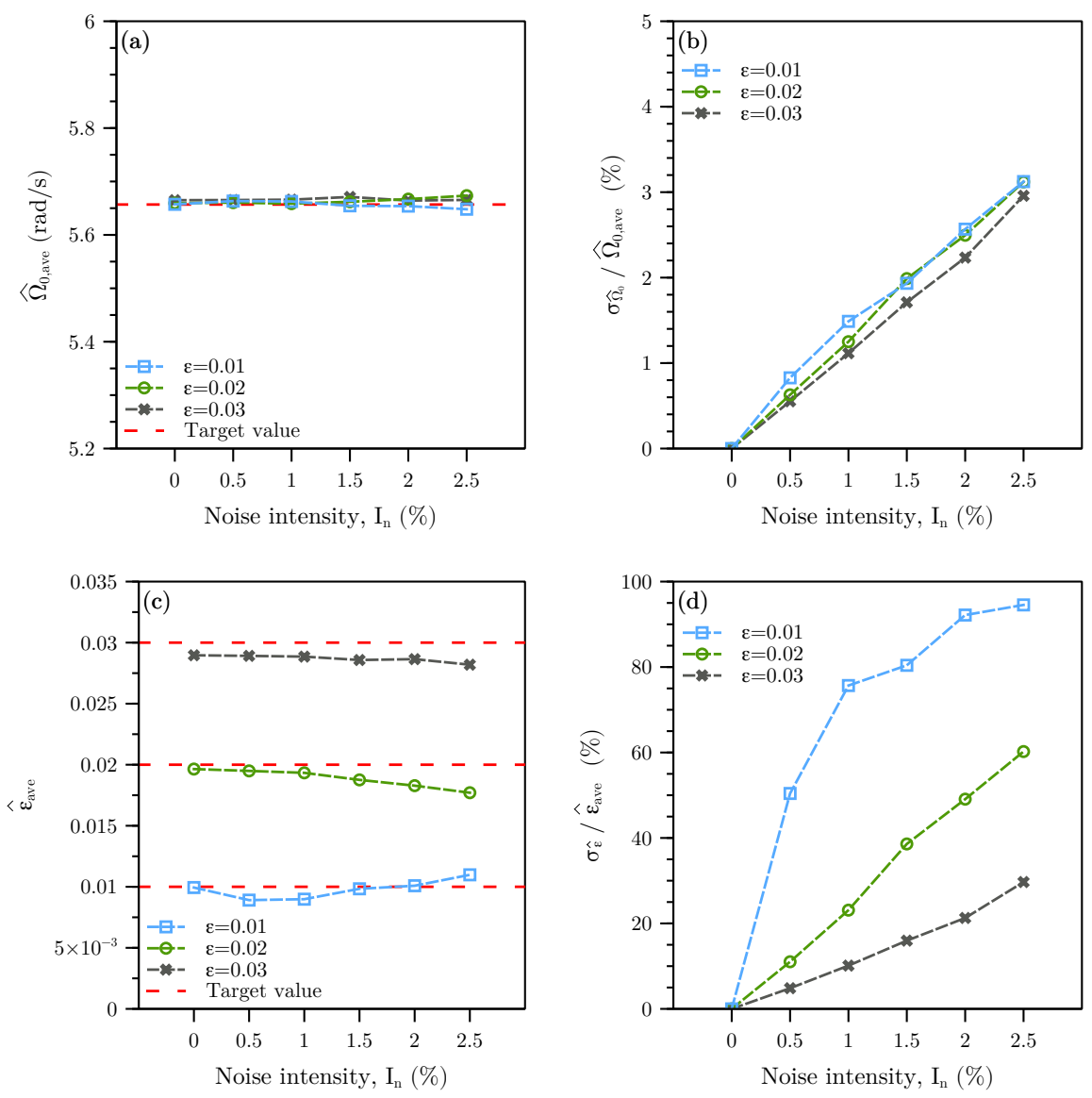

Figure 15: Results of the linear regression model, averaged over one-thousand runs, as a function of the noise intensity for a stay cable anchored to flexible restraints characterized by: $\Omega_{0}=5.66 \mathrm{rad} / \mathrm{s}, p=0.5$ and three different values of $\varepsilon: \varepsilon=0.01,0.02$ and 0.03 . The results have been obtained by setting the restraint parameter $p$ in Eq. (69) equal to: $p=0.5$. (a) Characteristic frequency $\Omega_{0}$ (target value: $\Omega_{0}=5.66 \mathrm{rad} / \mathrm{s}$ ). (b) Coefficient of variation of the estimated value of $\Omega_{0}$. (c) Non-dimensional bending stiffness $\varepsilon$ (target values: 0.01, 0.02 and 0.03 ).

(d) Coefficient of variation of the estimated value of $\varepsilon$. 
out to be only weakly affected by $\varepsilon$ (Figure 15(b)). This remark is consistent with the predictions of Eq. (74), characterized by a leading-order term independent of the cable non-dimensional bending stiffness. Figure 15(c) allows one to observe that the proposed identification procedure delivers average values of $\hat{\varepsilon}$ in very good agreement with the target ones (i.e. $\varepsilon=0.01,0.02$ and 0.03 ), for all values of noise intensity herein considered. Within this context, it is worth noting that the already mentioned modeling errors associated to the ideal condition of zero noise $\left(I_{n}=0\right)$ decrease for decreasing values of $\varepsilon$, being practically negligible for $\varepsilon=0.01$. The coefficient of variation $\sigma_{\hat{\varepsilon}} / \hat{\varepsilon}$ is shown in Figure 15(d) as a function of the noise intensity. It can be easily observed that, for a fixed value of noise intensity, $\sigma_{\hat{\varepsilon}} / \hat{\varepsilon}$ rapidly increases with the decrease of the non-dimensional bending stiffness $\varepsilon$.

These illustrations show that, at the limited extra cost of a coordinate transformation before the linear regression, we could develop a simple identification procedure that provides very good estimates of the cable tension, through $\hat{\Omega}_{0}$, with limited, controlled and rather noise-insensitive bias. At the same time, the method provides accurate estimates of the cable bending stiffness $\hat{\varepsilon}$ which are insensitive to the assumed flexibility in the boundary conditions.

\subsection{Comparisons among different identification procedures}

The outcomes of the two novel identification procedures proposed in Sections 3.2 (non-linear optimization problem) and 3.3 (linear regression model) are systematically compared, in the present Section, with reference to stay cables characterized by $\Omega_{0}=5.66 \mathrm{rad} / \mathrm{s}, p=0.5$ and three different values of $\varepsilon$, i.e.: $\varepsilon=0.01,0.02$ and 0.03 . The results of the classic approach relying on the taut string model (see Section 3.1) are also considered for comparison purposes.

The non-linear optimization problem (Section 3.2) has been solved by using the two variants of the DE algorithm already described in Section 4.1, i.e.: (i) the three-parameter scheme with unknown parameters $\Omega_{0}, \varepsilon$ and $p$ (curves labeled as "DE"), and (ii) the two-parameter scheme based on the pragmatical assumption $p=0.5$, with unknown parameters $\Omega_{0}$ and $\varepsilon$ (curves labeled as " $D E, p=0.5$ "). All control parameters of the DE algorithm are defined as explained in Section 4.1. Similarly as in Section 4.2, the linear regression model has been applied by considering three different assumptions to set the restraint parameter $p$ in Eq. (69): (i) $p=0$ (curves labeled as " $L R, p=0$ "), (ii) $p=0.5$ (" $L R, p=0.5 ")$, and (iii) $p=1$ (" $L R, p=1$ "). As already shown in Section 4.2, different assumptions on $p$ do not affect the estimate $\hat{\varepsilon}$ of the non-dimensional bending stiffness and its associated coefficient of variation $\sigma_{\hat{\varepsilon}} / \hat{\varepsilon}$. As a consequence, a single curve labeled as " $L R$ " is used to report the results of the linear regression model in terms of $\hat{\varepsilon}$ and $\sigma_{\hat{\varepsilon}} / \hat{\varepsilon}$. Two different variants of the taut string model have also been considered for comparison purposes, respectively based on the knowledge of: (i) the fundamental frequency of the cable only (curves labeled as "TS 1f"), and (ii) the five lower natural frequencies of the cable (curves labeled as "TS $5 f$ ").

Figures 16, 17 and 18 show the results of the different identification algorithms, averaged over the number of runs $N R=1000$ considered for each noise intensity level. Figures 16-18 (a) report the average values of $\hat{\Omega}_{0}$. The results of the two different variants of the DE algorithm (" $D E$ " and " $D E, p=0.5$ ") are practically coincident with the ones of the linear regression model " $L R, p=0.5$ ". The results of the taut string model "TS $1 f$ ", on the other hand, turns out to be very close to the ones of the linear regression model " $L R, p=0$ ". This was expected, since both models neglect the effect of the rotational stiffness of the anchoring devices. Minimum and maximum values of $\hat{\Omega}_{0}$ are systematically delivered by, respectively the " $L R, p=1$ " and the "TS $5 f$ " models. The difference between the two taut string models " $T S 1 f$ " and "TS $5 f$ " can be easily explained by recalling that, for these procedures, the bias of the estimator $\hat{\Omega}_{0}$ increase with the increase of the number of modes considered for identification purposes, see Eq. (47).

Figures 16-18 (b) show the coefficient of variation $\sigma_{\hat{\Omega}_{0}} / \hat{\Omega}_{0}$ as a function of the noise intensity $I_{n}$. All identification strategies, with the only exception of the three-parameter identification algorithm (" $D E$ "), are characterized by a somewhat linear relation between $\sigma_{\hat{\Omega}_{0}} / \hat{\Omega}_{0}$ and $I_{n}$, with slope values that are: (a) strictly independent of $\varepsilon$ for the identification strategies " $L R, p=0$ ", "TS $1 f$ " and "TS $5 f$ ", (b) substantially independent of $\varepsilon$ for the remaining identification strategies (also see the discussion in Sections 4.1 and 4.2). This distinction follows the motivation that a 

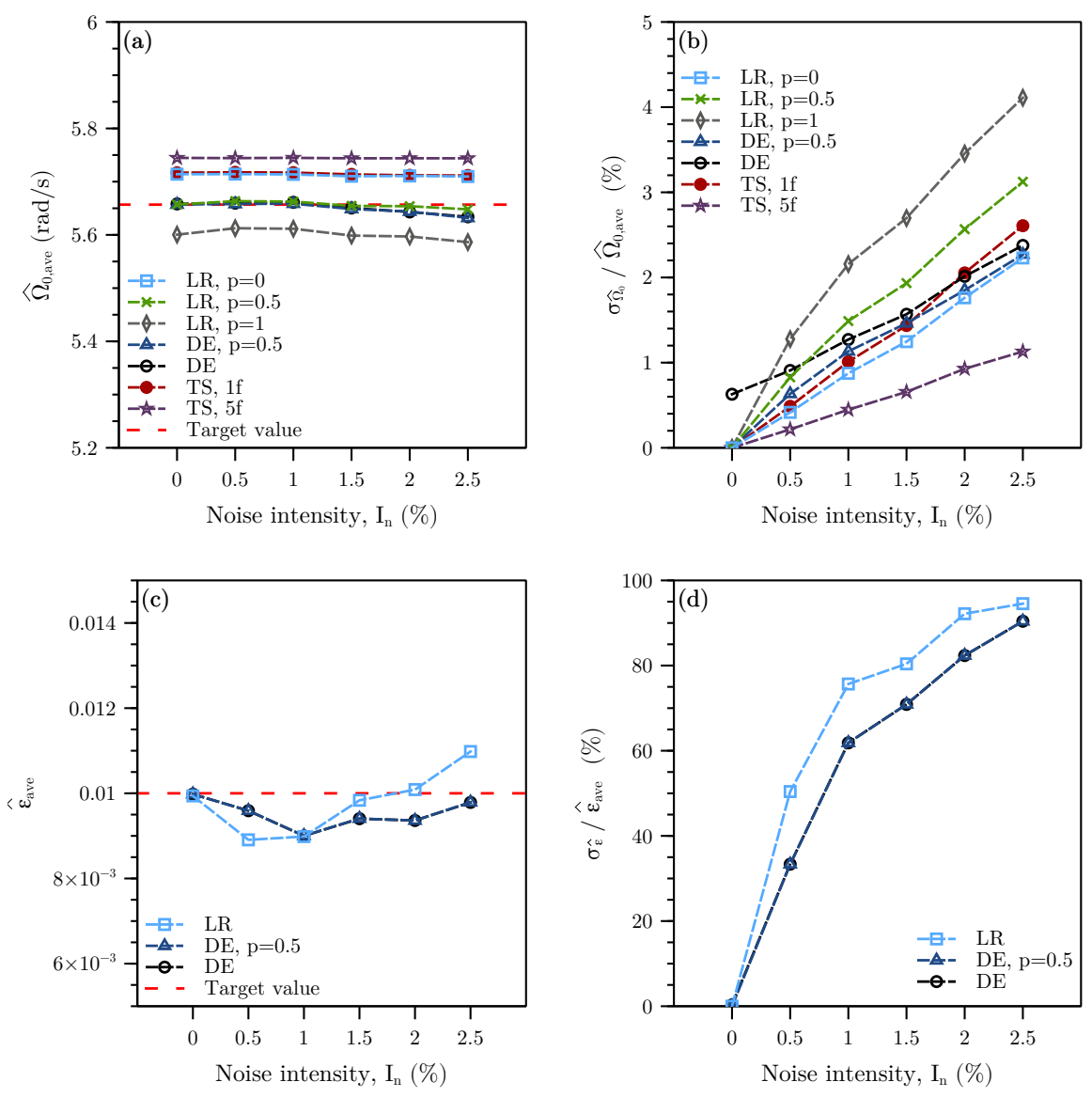

Figure 16: Comparison among different identification procedures. Target values are $\Omega_{0}=5.66 \mathrm{rad} / \mathrm{s}, \varepsilon=0.01$, $p=0.5$.

feature of a robust identification strategy should be such that a discrepancy on the estimation of $\varepsilon$ shall not spoils the quality of the estimation of $\Omega_{0}$ and, hence, $T$. Minimum and maximum slope values are respectively associated to the "TS $5 f$ " and " $L R, p=1$ " models.

Figures 16-18 (c) easily allow one to appreciate that both DE algorithms (i.e. " $D E$ " and " $D E$, $p=0.5 ")$ give average values of $\hat{\varepsilon}$ very close to the ones of the more simple linear regression model, for any value of the noise intensity herein considered. On the other hand, DE algorithms estimates are characterized, in general, by smaller values of the coefficient of variation $\sigma_{\hat{\varepsilon}} / \hat{\varepsilon}$, as it can be observed from Figures 16-18 (d).

\section{Conclusions}

Starting from the mechanical model of a cable with a small bending stiffness and flexible anchorages in both translation and rotation, two different identification strategies have been developed. These identification methods provide estimates of the cable axial force $T$ and of the (small) dimensionless bending stiffness $\varepsilon$ while considering the end restraints as unknown parameter. They rely on the asymptotic expansions of the natural frequencies of such a cable for small bending stiffness, which is typical of stay cables and structural elements with similar aspect ratios.

Indeed, as a prelude to the derivation of the identification procedure, we have shown that, up to the third order in $\varepsilon$, the natural frequencies of the cable are only affected by three parameters, $\Omega_{0}, \varepsilon$ and $p$. The first two are related to the axial force in the cable (which is the sole parameter affecting the natural frequencies at leading order) and its bending stiffness while the latter is a 

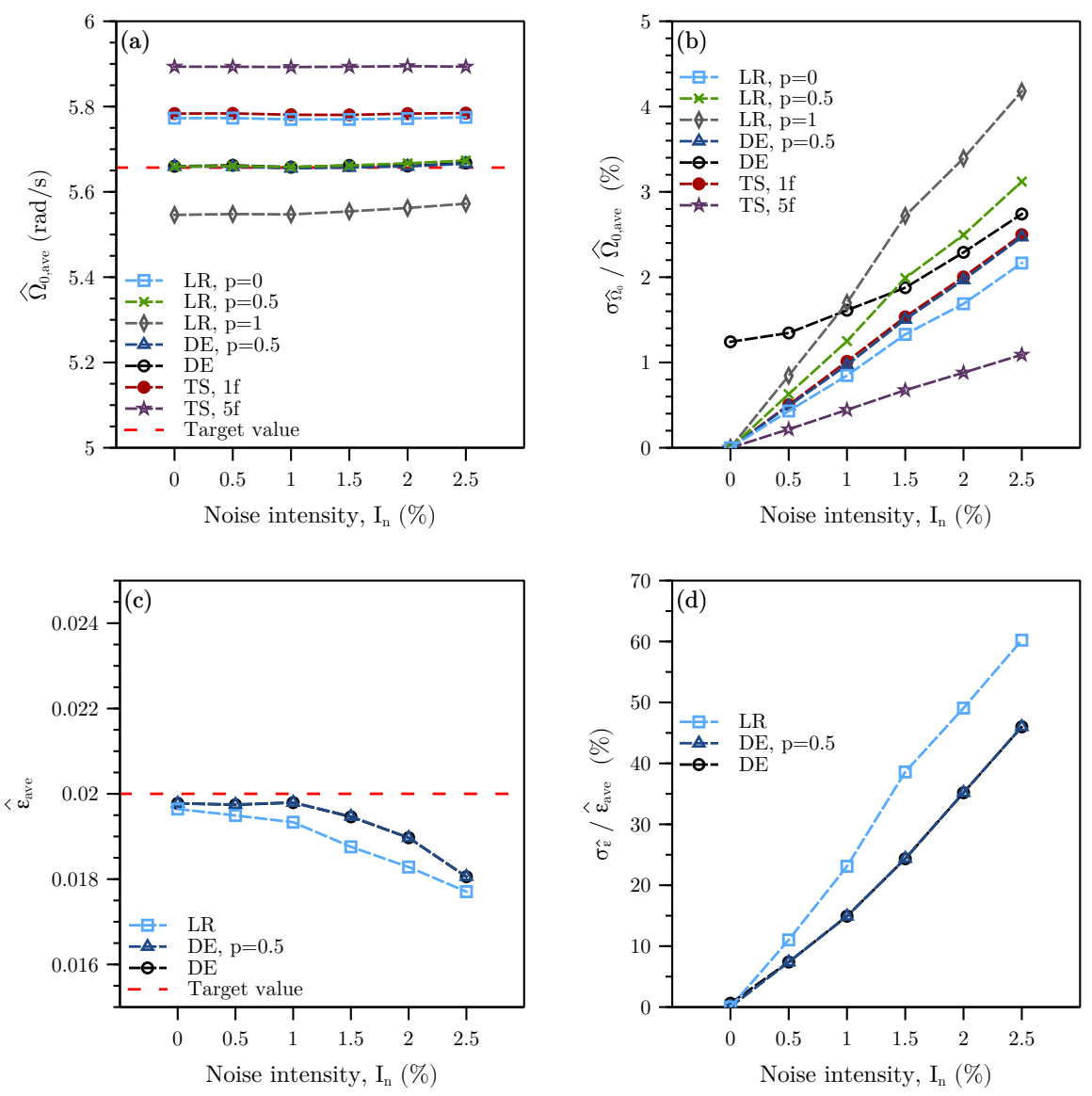

Figure 17: Comparison among different identification procedures. Target values are $\Omega_{0}=5.66 \mathrm{rad} / \mathrm{s}, \varepsilon=0.02$, $p=0.5$. 

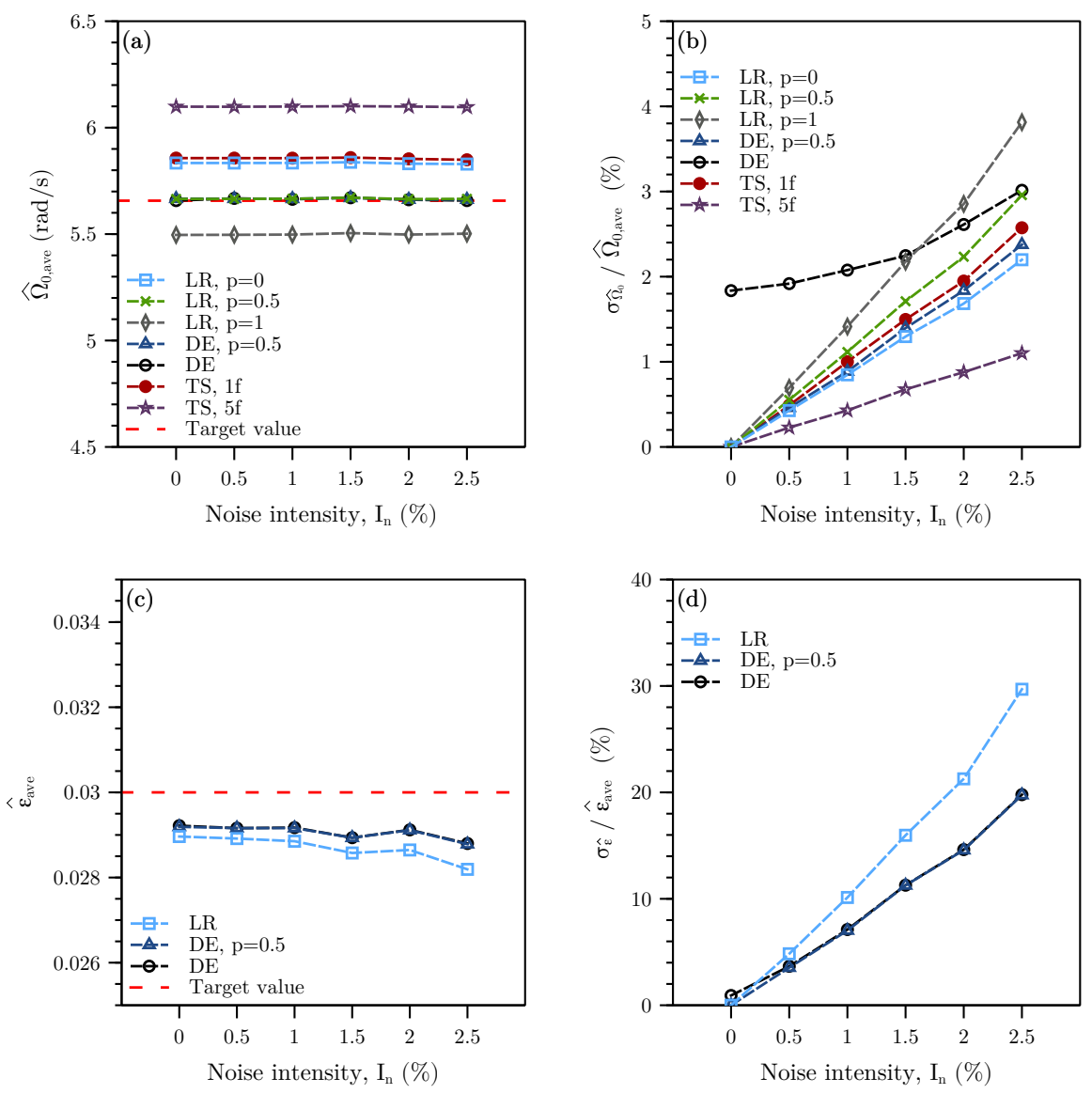

Figure 18: Comparison among different identification procedures. Target values are $\Omega_{0}=5.66 \mathrm{rad} / \mathrm{s}, \varepsilon=0.03$, $p=0.5$. 
dimensionless group translating the influence of boundary restraints. Since they all act through this unique parameter, it is therefore impossible to separate, on the sole basis of natural frequencies, the influence of translational and rotational flexibilities of anchorages, for vanishingly small bending stiffness.

A first identification procedure naturally relies upon a constrained least-square optimization problem, minimizing a norm of the difference between the measured natural frequencies of a cable and those predicted by the asymptotic model. This optimization problem is solved with the Differential Evolution algorithm which is based on the evolution of a swarm of best candidates, non gradient-based and capable of dealing with several local minima. It has been implemented in two versions, a three-dimensional one aiming at identifying $\Omega_{0}, \varepsilon$ and $p$ and a two-dimensional one aiming at identifying $\Omega_{0}$ and $\varepsilon$, while $p$ is imposed.

The second identification procedure is based on a linear regression in a transformed coordinate system. The proposed identification method is as simple as the common method based on the taut string model. It is also a straightforward generalization of the methods based on the fitting of a two-parameter model (including the equivalent of $\Omega_{0}$ and $\varepsilon$ ), with the major difference that the management of boundary conditions are herein explicitly taken into consideration. In the unlikely case of known rotational and translational anchorage rigidities, parameter $p$ can be imposed and this yields the best performances in the proposed method. Otherwise, it is suggested to set $p$ to its median value $p=0.5$ in order to limit the undesired influence of unknown boundary conditions. Any other user-defined choice is also possible, including an interval analysis.

The quality of the results obtained with the two proposed identification methods is assessed by means of the statistics of the bias and dispersion of the estimators $\hat{\Omega}_{0}$ and $\hat{\varepsilon}$. These are compared to those obtained with the standard taut-string model. In short, it is shown that the linear regression model performs as good as the first identification procedure which is based on the Differential Evolution solver and much more computationally demanding. Compared to the taut string model, the proposed linear regression approach provides a very similar estimate of the cable axial force but, additionally and with almost no extra cost, an estimate of the cable bending stiffness. Furthermore, the influence of the stiffness of anchorages is fully controlled through parameter $p$. The simplicity of the proposed formulation also made it possible to derive analytical expressions for the uncertainty propagation analysis which gives a clear picture of the way parameters influence the quality of the identification procedure.

Finally, since it generalizes many particular cases, the proposed method can be used as such in the various contexts today where cable tension and bending stiffness need to be identified with the sole knowledge of measured natural frequencies. 


\section{Appendix A: Components of the boundary condition matrix B}

The components of the boundary condition matrix B (see Eq. (21) for definition) will be denoted in the following as $B_{i, j}(i, j=1, \ldots, 4)$, with subscripts $i$ and $j$ identifying, respectively, the $i^{t h}$ row and the $j^{\text {th }}$ column of the matrix. Components $B_{i, j}$ are, in general, functions of the nondimensional frequency $\omega$ and of the parameters: $\mathscr{P}=\left\{\varepsilon, \rho_{T 0}, \rho_{T 1}, \rho_{R 0}, \rho_{R 1}\right\}$. In order to avoid cumbersome expressions, however, Eqs. (A.1)-(A.16) are written by exploiting the definition of the variables $z_{j}=z_{j}(\omega)(j=1,2)$ introduced in Eq. (20) and without making explicit the functional dependence on $\omega$.

$$
\begin{aligned}
& B_{1,1}=-\left(1-\rho_{T 0}\right)\left(1+\varepsilon^{2} z_{1}^{2}\right) \varepsilon z_{1} \\
& B_{1,2}=\rho_{T 0} \\
& B_{1,3}=\rho_{T 0}+\left(1-\rho_{T 0}\right)\left(1-\varepsilon^{2} z_{2}^{2}\right) \varepsilon z_{2} \\
& B_{1,4}=\left(\rho_{T 0}-\left(1-\rho_{T 0}\right)\left(1-\varepsilon^{2} z_{2}^{2}\right) \varepsilon z_{2}\right) \exp \left(-z_{2}\right) \\
& B_{2,1}=-\rho_{T 1} \sin \left(z_{1}\right)-\left(1-\rho_{T 1}\right) \cos \left(z_{1}\right)\left(1+\varepsilon^{2} z_{1}^{2}\right) \varepsilon z_{1} \\
& B_{2,2}=-\rho_{T 1} \cos \left(z_{1}\right)+\left(1-\rho_{T 1}\right) \sin \left(z_{1}\right)\left(1+\varepsilon^{2} z_{1}^{2}\right) \varepsilon z_{1} \\
& B_{2,3}=\left(-\rho_{T 1}+\left(1-\rho_{T 1}\right)\left(1-\varepsilon^{2} z_{2}^{2}\right) \varepsilon z_{2}\right) \exp \left(-z_{2}\right) \\
& B_{2,4}=-\rho_{T 1}-\left(1-\rho_{T 1}\right)\left(1-\varepsilon^{2} z_{2}^{2}\right) \varepsilon z_{2} \\
& B_{3,1}=-\varepsilon z_{1} \rho_{R 0} \\
& B_{3,2}=-\left(1-\rho_{R 0}\right) \varepsilon^{2} z_{1}^{2} \\
& B_{3,3}=\left(\rho_{R 0}+\left(1-\rho_{R 0}\right) \varepsilon z_{2}\right) \varepsilon z_{2} \\
& B_{3,4}=\left(-\rho_{R 0}+\left(1-\rho_{R 0}\right) \varepsilon z_{2}\right) \varepsilon z_{2} \exp \left(-z_{2}\right) \\
& B_{4,1}=\left(\rho_{R 1} \cos \left(z_{1}\right)-\left(1-\rho_{R 1}\right) \sin \left(z_{1}\right) \varepsilon z_{1}\right) \varepsilon z_{1} \\
& B_{4,2}=\left(-\rho_{R 1} \sin \left(z_{1}\right)-\left(1-\rho_{R 1}\right) \cos \left(z_{1}\right) \varepsilon z_{1}\right) \varepsilon z_{1} \\
& B_{4,3}=\left(-\rho_{R 1}+\left(1-\rho_{R 1}\right) \varepsilon z_{2}\right) \varepsilon z_{2} \exp \left(-z_{2}\right) \\
& B_{4,4}=\left(\rho_{R 1}+\left(1-\rho_{R 1}\right) \varepsilon z_{2}\right) \varepsilon z_{2}
\end{aligned}
$$




\section{Appendix B: Components of matrices $\mathrm{B}_{(0)}, \mathrm{B}_{(1)}$ and $\mathrm{B}_{(2)}$}

The components of matrices $\mathbf{B}_{(0)}, \mathbf{B}_{(1)}$ and $\mathbf{B}_{(2)}$ (see Eq. (34) for definition) will be respectively denoted as $B_{(0) i, j}, B_{(1) i, j}$ and $B_{(2) i, j}(i, j=1, \ldots, 4)$, with the same notation adopted in Appendix A.

All non-zero components of the matrix $\mathbf{B}_{(0)}$ are reported in the following Eqs. (B.1)-(B.7):

$$
\begin{gathered}
B_{(0) 1,2}=\rho_{T 0} \\
B_{(0) 1,3}=\rho_{T 0} \\
B_{(0) 2,1}=-\rho_{T 1} \sin \left(\omega_{(0)}\right) \\
B_{(0) 2,2}=-\rho_{T 1} \cos \left(\omega_{(0)}\right) \\
B_{(0) 2,4}=-\rho_{T 1} \\
B_{(0) 3,3}=1 \\
B_{(0) 4,4}=1
\end{gathered}
$$

All non-zero components of the matrix $\mathbf{B}_{(1)}$ are reported in the following Eqs. (B.8)-(B.13):

$$
\begin{gathered}
B_{(1) 1,1}=-\omega_{(0)}\left(1-\rho_{T 0}\right) \\
B_{(1) 2,1}=-\omega_{(0)} \cos \left(\omega_{(0)}\right)\left(1-\rho_{T 1}\right)-\omega_{(1)} \cos \left(\omega_{(0)}\right) \rho_{T 1} \\
B_{(1) 2,2}=\omega_{(0)} \sin \left(\omega_{(0)}\right)\left(1-\rho_{T 1}\right)+\omega_{(1)} \sin \left(\omega_{(0)}\right) \rho_{T 1} \\
B_{(1) 3,1}=-\omega_{(0)} \rho_{R 0} \\
B_{(1) 4,1}=\omega_{(0)} \cos \left(\omega_{(0)}\right) \rho_{R 1} \\
B_{(1) 4,2}=-\omega_{(0)} \sin \left(\omega_{(0)}\right) \rho_{R 1}
\end{gathered}
$$

All non-zero components of the matrix $\mathbf{B}_{(2)}$ are reported in the following Eqs. (B.14)-(B.24):

$$
\begin{gathered}
B_{(2) 1,1}=-\omega_{(1)}\left(1-\rho_{T 0}\right) \\
B_{(2) 1,3}=-\omega_{(0)}^{2}\left(1-\rho_{T 0}\right) \\
B_{(2) 2,1}=\frac{1}{2} \omega_{(0)}^{3} \cos \left(\omega_{(0)}\right) \rho_{T 1}+\frac{1}{2} \omega_{(0)}^{2} \sin \left(\omega_{(0)}\right) \rho_{T 1} \\
+\omega_{(1)} \omega_{(0)} \sin \left(\omega_{(0)}\right)\left(1-\rho_{T 1}\right) \\
-\omega_{(1)} \cos \left(\omega_{(0)}\right)\left(1-\rho_{T 1}\right)-\omega_{(2)} \cos \left(\omega_{(0)}\right) \rho_{T 1} \\
B_{(2) 2,2}=-\frac{1}{2} \omega_{(0)}^{3} \sin \left(\omega_{(0)}\right) \rho_{T 1}+\frac{1}{2} \omega_{(1)}^{2} \cos \left(\omega_{(0)}\right) \rho_{T 1} \\
+\omega_{(1)} \omega_{(0)} \cos \left(\omega_{(0)}\right)\left(1-\rho_{T 1}\right) \\
+\omega_{(1)} \sin \left(\omega_{(0)}\right)\left(1-\rho_{T 1}\right)+\omega_{(2)} \sin \left(\omega_{(0)}\right) \rho_{T 1}
\end{gathered}
$$




$$
\begin{gathered}
B_{(2) 2,4}=\omega_{(0)}^{2}\left(1-\rho_{T 1}\right) \\
B_{(2) 3,1}=-\omega_{(1)} \rho_{R 0} \\
B_{(2) 3,2}=-\omega_{(0)}^{2}\left(1-\rho_{R 0}\right) \\
B_{(2) 3,3}=\omega_{(0)}^{2}\left(1-\frac{1}{2} \rho_{R 0}\right) \\
B_{(2) 4,1}=-\omega_{(0)}^{2} \sin \left(\omega_{(0)}\right)\left(1-\rho_{R 1}\right) \\
-\omega_{(1)} \omega_{(0)} \sin \left(\omega_{(0)}\right) \rho_{R 1}+\omega_{(1)} \cos \left(\omega_{(0)}\right) \rho_{R 1} \\
B_{(2) 4,2}=-\omega_{(0)}^{2} \cos \left(\omega_{(0)}\right)\left(1-\rho_{R 1}\right) \\
-\omega_{(1)} \omega_{(0)} \cos \left(\omega_{(0)}\right) \rho_{R 1}-\omega_{(1)} \sin \left(\omega_{(0)}\right) \rho_{R 1} \\
B_{(2) 4,4}=\omega_{(0)}^{2}\left(1-\frac{1}{2} \rho_{R 1}\right)
\end{gathered}
$$

\section{Appendix C: Solution of the system of equations (36)}

In this Appendix, the system of Eqs. (36) is fully solved for the coefficients $\omega_{(i)}$ and $\boldsymbol{\alpha}_{(i)}$ $(i=0,1,2)$ of the second-order accurate asymptotic expansions of the eigenvalues $\omega$ (Eq. (32)) and eigenvectors $\boldsymbol{\alpha}$ (Eq. (33)) of problem (21). The solution is sought through a cascaded approach, starting from the leading order problem (i.e. $\left.\operatorname{ord}\left(\varepsilon^{0}\right)\right)$ in $(36)$ and moving towards the higher order ones.

\section{Leading order solution}

The solution of the leading order problem (i.e. $\left.\operatorname{ord}\left(\varepsilon^{0}\right)\right)$ in $(36)$ amounts to find the the eigenvalues and eigenvectors of the matrix $\mathbf{B}_{(0)}$, whose components are defined in the Appendix B (see Eqs. (B.1)-(B.7)).

It can be easily verified that the eigenvalues of $\mathbf{B}_{(0)}$ coincide, as expected, with the nondimensional natural frequencies of the taut string model, i.e. (cf. Eq. (18)):

$$
\omega_{(0) k}=k \pi, k \in \mathbb{N}^{+}
$$

The right $\left(\boldsymbol{\alpha}_{(0) k}\right)$ and left $\left(\boldsymbol{\beta}_{(0) k}^{T}\right)$ eigenvectors of $\mathbf{B}_{(0)}$ can be expressed as:

$$
\begin{gathered}
\boldsymbol{\alpha}_{(0) k}=\boldsymbol{\alpha}_{0}=(1,0,0,0)^{T}, \forall k \in \mathbb{N}^{+} \\
\boldsymbol{\beta}_{(0) k}^{T}=\left(1,(-1)^{k} \frac{\rho_{T 0}}{\rho_{T 1}},-\rho_{T 0},(-1)^{k} \rho_{T 0}\right), k \in \mathbb{N}^{+}
\end{gathered}
$$


951 whose solutions for $\boldsymbol{\alpha}_{(2) k}$ read:

$$
\boldsymbol{\alpha}_{(1) k}=k \pi\left(0,-p_{0} p, \rho_{R 0} p,(-1)^{k+1} \rho_{R 1} p\right)^{T}
$$

First order correction

can be pre-multiplied by $\boldsymbol{\beta}_{(0) k}^{T}$ yielding the scalar equation:

$$
\boldsymbol{\beta}_{(0) k}^{T} \mathbf{B}_{(1)}\left(\omega_{(1) k}, \omega_{(0) k}\right) \boldsymbol{\alpha}_{0}=0
$$

Solutions of Eq. (18) for $\omega_{(1) k}$ read:

$$
\omega_{(1) k}=2 p k \pi, \forall k \in \mathbb{N}^{+}
$$

where $p$ is the restraint parameter defined in Eq. (26). By substituting Eqs. (C.1), (C.2) and (C.5) in the second equation of (36), then, one can get the linear equation:

$$
\mathbf{B}_{(0)}\left(\omega_{(0) k}\right) \boldsymbol{\alpha}_{(1) k}=-\mathbf{B}_{(1)}\left(\omega_{(1) k}, \omega_{(0) k}\right) \boldsymbol{\alpha}_{0}
$$

whose solutions for $\boldsymbol{\alpha}_{(1) k}$ read:

$$
\boldsymbol{\alpha}_{(1) k}=k \pi\left(0,-p_{0}, \rho_{R 0},(-1)^{k+1} \rho_{R 1}\right)^{T}
$$

with the definition:

$$
p_{0}=1+\rho_{R 0}-\frac{1}{\rho_{T 0}}, \rho_{T 0}>0
$$

\section{Second order correction}

Once both the leading order solution and the first order correction are known, the row corresponding to the $\operatorname{ord}\left(\varepsilon^{2}\right)$ problem in (36) can be pre-multiplied by $\boldsymbol{\beta}_{(0) k}^{T}$ yielding the scalar equation:

$$
\boldsymbol{\beta}_{(0) k}^{T} \mathbf{B}_{(1)}\left(\omega_{(1) k}, \omega_{(0) k}\right) \boldsymbol{\alpha}_{(1) k}+\boldsymbol{\beta}_{(0) k}^{T} \mathbf{B}_{(2)}\left(\omega_{(2) k}, \omega_{(1) k}, \omega_{(0) k}\right) \boldsymbol{\alpha}_{0}=0
$$

Solutions of Eq. (C.9) for $\omega_{(2) k}$ read:

$$
\omega_{(2) k}=\left(\frac{(k \pi)^{2}}{2}+4 p^{2}\right), k \pi \forall k \in \mathbb{N}^{+}
$$

where $p$ is the restraint parameter defined in Eq. (26). By substituting Eqs. (C.1), (C.2), (C.5), (C.7) and (C.10) in the third equation of (36), then, one can get the linear equation:

$$
\mathbf{B}_{(0)}\left(\omega_{(0) k}\right) \boldsymbol{\alpha}_{(2) k}=-\mathbf{B}_{(1)}\left(\omega_{(1) k}, \omega_{(0) k}\right) \boldsymbol{\alpha}_{(1) k}-\mathbf{B}_{(2)}\left(\omega_{(2) k}, \omega_{(1) k}, \omega_{(0) k}\right) \boldsymbol{\alpha}_{0}
$$

Acknowledgments

The project is funded by the Wallonia Public Service. It is carried out by the University of Liège and the company V2i (www.v2i.be). M.G. acknowledges the support of the FNRS (Belgian Fund for Scientific Research). 


\section{References}

[1] A. Arena, A. Pacitti, and W. Lacarbonara. Nonlinear response of elastic cables with flexuraltorsional stiffness. International journal of solids and structures, 87:267-277, 2016.

[2] C. Bedon, M. Dilena, and A. Morassi. Ambient vibration testing and structural identification of a cable-stayed bridge. Meccanica, 51(11):2777-2796, 2016.

[3] S. Benecke and J.H. Van Vuuren. Modelling torsion in an elastic cable in space. Applied mathematical modelling, 29(2):117-136, 2005.

[4] F. Benedettini and C. Gentile. Operational modal testing and fe model tuning of a cablestayed bridge. Engineering Structures, 33(6):2063-2073, 2011.

[5] Bureau International des Poids et Mesures BIPM. Evaluation of measurement data - guide to the expression of uncertainty in measurements. Working Group 1 of the Joint Committee for Guides in Metrology (JSGM/WG 1), 2008.

[6] E. Caetano. Cable vibrations in cable-stayed bridges, volume 9. IABSE, 2007.

[7] E. Caetano and A. Cunha. Dynamic testing of cable structures. In MATEC Web of Conferences, volume 24, page 01002. EDP Sciences, 2015.

[8] E. Caetano, R. Bartek, F. Magalhães, C. Keenan, and G. Trippick. Assessment of cable forces at the london 2012 olympic stadium roof. Structural Engineering International, 23(4): 489-500, 2013.

[9] M.A. Ceballos and C.A. Prato. Determination of the axial force on stay cables accounting for their bending stiffness and rotational end restraints by free vibration tests. Journal of Sound and Vibration, 317(1-2):127-141, 2008.

[10] C.C. Chen, W.H. Wu, S.Y. Chen, and G. Lai. A novel tension estimation approach for elastic cables by elimination of complex boundary condition effects employing mode shape functions. Engineering Structures, 166:152-166, 2018.

[11] S. Cho, J. Yim, S.W. Shin, H.J. Jung, C.B. Yun, and M.L. Wang. Comparative field study of cable tension measurement for a cable-stayed bridge. Journal of Bridge Engineering, 18 (8):748-757, 2013.

[12] A. Cunha, E. Caetano, and R. Delgado. Dynamic tests on large cable-stayed bridge. Journal of Bridge Engineering, 6(1):54-62, 2001.

[13] A. Cunha, E. Caetano, F. Magalhães, and C. Moutinho. Recent perspectives in dynamic testing and monitoring of bridges. Structural Control and Health Monitoring, 20(6):853-877, 2013.

[14] S. Das and P. N. Suganthan. Differential evolution: A survey of the state-of-the-art. IEEE transactions on evolutionary computation, 15(1):4-31, 2010.

[15] S. Das, A. Abraham, U.K. Chakraborty, and A. Konar. Differential evolution using a neighborhood-based mutation operator. IEEE Transactions on Evolutionary Computation, 13(3):526-553, 2009.

[16] S. Das, S.S. Mullick, and P.N. Suganthan. Recent advances in differential evolution-an updated survey. Swarm and Evolutionary Computation, 27:1-30, 2016.

[17] P. de Mars and D. Hardy. Mesure des efforts dans les structures a cables. Annales TP Belgique, 6:515-531, 1985. 
[18] V. Denoël and T. Canor. Patching asymptotics solution of a cable with a small bending stiffness. Journal of Structural Engineering, 139(2):180-187, 2013.

[19] V. Denoël and E. Detournay. Multiple scales solution for a beam with a small bending stiffness. Journal of engineering mechanics, 136(1):69-77, 2010.

[20] M. Domaneschi, M.P. Limongelli, and L. Martinelli. Damage detection and localization on a benchmark cable-stayed bridge. Earthquakes and Structures, 8(5):1113-1126, 2015.

[21] F. Foti and L. Martinelli. Mechanical modeling of metallic strands subjected to tension, torsion and bending. International Journal of Solids and Structures, 91:1-17, 2016.

[22] F. Foti and L. Martinelli. Finite element modeling of cable galloping vibrations -part i: Formulation of mechanical and aerodynamic co-rotational elements. Archive of Applied Mechanics, 88(5):645-670, 2018.

[23] F. Foti, L. Martinelli, and F. Perotti. Numerical integration of the equations of motion of structural systems undergoing large 3d rotations: dynamics of corotational slender beam elements. Meccanica, 50(3):751-765, 2015.

[24] R. Geier, G. De Roeck, and R. Flesch. Accurate cable force determination using ambient vibration measurements. Structure and Infrastructure Engineering, 2(1):43-52, 2006.

[25] C. Gentile. Deflection measurement on vibrating stay cables by non-contact microwave interferometer. NDT \& E International, 43(3):231-240, 2010.

[26] M. Géradin and D. Rixen. Mechanical Vibrations: Theory and Application to Structural Dynamics. Wiley, 1999. ISBN 9780471975465.

[27] E.J. Hinch. Perturbation Methods. Cambridge Texts in Applied Mathematics. Cambridge University Press, 1991.

[28] Y.H. Huang, J.Y. Fu, R.H. Wang, Q. Gan, and A.R. Liu. Unified practical formulas for vibration-based method of cable tension estimation. Advances in Structural Engineering, 18 (3):405-422, 2015.

[29] R.A. Ibrahim. Nonlinear vibrations of suspended cables - part iii: random excitation and interaction with fluid flow. Appl. Mech. Rev., 57(6):515-549, 2004.

[30] H. M. Irvine and T.K. Caughey. The linear theory of free vibrations of a suspended cable. Proceedings of the Royal Society of London. A. Mathematical and Physical Sciences, 341 (1626):299-315, 1974.

[31] H.M. Irvine. Cable structures. mit press series in structural mechanics. 1981.

[32] J. Irving and N. Mullineux. Mathematics in physics and engineering. Academic Press New York, 1959.

[33] T. Kernicky, M. Whelan, and E. Al-Shaer. Dynamic identification of axial force and boundary restraints in tie rods and cables with uncertainty quantification using set inversion via interval analysis. Journal of Sound and Vibration, 423:401-420, 2018.

[34] B.H. Kim and T. Park. Estimation of cable tension force using the frequency-based system identification method. Journal of sound and Vibration, 304(3-5):660-676, 2007.

[35] B.H. Kim, T. Park, H. Shin, and T.Y. Yoon. A comparative study of the tension estimation methods for cable supported bridges. International Journal of Steel Structures, 7(1):77-84, 2007. 
[36] J.M. Ko, Y.Q. Ni, H.F. Zhou, J.Y. Wang, and X.T. Zhou. Investigation concerning structural health monitoring of an instrumented cable-stayed bridge. Structures $\mathcal{G}$ Infrastructure Engineering, 5(6):497-513, 2009.

[37] W. Lacarbonara, A. Paolone, and F. Vestroni. Elastodynamics of nonshallow suspended cables: linear modal properties. Journal of Vibration and Acoustics, 129:425-433, 2007.

[38] W. Lacarbonara, A. Paolone, and F. Vestroni. Non-linear modal properties of non-shallow cables. International Journal of Non-Linear Mechanics, 42(3):542-554, 2007.

[39] M. Lepidi, V. Gattulli, and F. Vestroni. Damage identification in elastic suspended cables through frequency measurement. Journal of Vibration and Control, 15(6):867-896, 2009.

[40] E. Lofrano, A. Paolone, and M. Vasta. Identification of uncertain vibrating beams through a perturbation approach. ASCE-ASME Journal of Risk and Uncertainty in Engineering Systems, Part A: Civil Engineering, 2(2):C4015006, 2016.

[41] A. Luongo, D. Zulli, and G. Piccardo. Analytical and numerical approaches to nonlinear galloping of internally resonant suspended cables. Journal of Sound and Vibration, 315(3): 375-393, 2008.

[42] L. Ma. A highly precise frequency-based method for estimating the tension of an inclined cable with unknown boundary conditions. Journal of Sound and Vibration, 409:65-80, 2017.

[43] K.G. McConnell and C.N. Chang. A study of the axial-torsional coupling effect on a sagged transmission line. Experimental Mechanics, 26(4):324-329, 1986.

[44] A.B. Mehrabi. In-service evaluation of cable-stayed bridges, overview of available methods and findings. Journal of Bridge Engineering, 11(6):716-724, 2006.

[45] A.B. Mehrabi and H. Tabatabai. Unified finite difference formulation for free vibration of cables. Journal of Structural Engineering, 124(11):1313-1322, 1998.

[46] P.M. Morse and K.U. Ingard. Theoretical acoustics. International Series in Pure and Applied Physics. McGraw-Hill, 1968.

[47] A. Pacitti, M. Peigney, F. Bourquin, and W. Lacarbonara. Experimental data based cable tension identification via nonlinear static inverse problem. Procedia engineering, 199:453-458, 2017.

[48] A. Papoulis and S.U. Pillai. Probability, random variables, and stochastic processes. Tata McGraw-Hill Education, 2002.

[49] A.G. Piersol and T.L. Paez. Harris' shock and vibration handbook, sixth edition. 2010.

[50] G. Rega. Nonlinear vibrations of suspended cables - part i: Modeling and analysis. Appl. Mech. Rev., 57(6):443-478, 2004.

[51] G. Rega. Nonlinear vibrations of suspended cables - part ii: deterministic phenomena. Appl. Mech. Rev., 57(6):479-514, 2004.

[52] João P Santos, Christian Crémona, Luís Calado, Paulo Silveira, and André D Orcesi. On-line unsupervised detection of early damage. Structural Control and Health Monitoring, 23(7): 1047-1069, 2016.

[53] R. Storn and K. Price. Differential evolution - a simple and efficient heuristic for global optimization over continuous spaces. Journal of global optimization, 11(4):341-359, 1997.

[54] P. Van Overschee and B. De Moor. Subspace algorithms for the stochastic identification problem. Automatica, 29(3):649-660, 1993. 
[55] A.B. Vasil'eva. Asymptotic behaviour of solutions to certain problems involving non-linear differential equations containing a small parameter multiplying the highest derivatives. Russian Mathematical Surveys, 18(3):13, 1963.

[56] P. Villaggio. Mathematical models for elastic structures. Cambridge University Press, 2005.

[57] S. Weisberg. Applied linear regression. John Wiley \& Sons, 2005.

[58] Wen-Hwa Wu, Sheng-Wei Wang, Chien-Chou Chen, and Gwolong Lai. Application of stochastic subspace identification for stay cables with an alternative stabilization diagram and hierarchical sifting process. Structural Control and Health Monitoring, 23(9):1194-1213, 2016.

[59] B. Yan, W. Chen, J. Yu, and X. Jiang. Mode shape-aided tension force estimation of cable with arbitrary boundary conditions. Journal of Sound and Vibration, 440:315-331, 2019.

[60] S. Zhang, R. Shen, Y. Wang, G. De Roeck, G. Lombaert, and K. Dai. A two-step methodology for cable force identification. Journal of Sound and Vibration, 472:115201, 2020.

[61] K. Zielinski and R. Laur. Stopping criteria for differential evolution in constrained singleobjective optimization. In Advances in differential evolution, pages 111-138. Springer, 2008.

[62] H. Zui, T. Shinke, and Y. Namita. Practical formulas for estimation of cable tension by vibration method. Journal of structural engineering, 122(6):651-656, 1996. 